BULLETIN (New Series) OF THE

AMERICAN MATHEMATICAL SOCIETY

Volume 38, Number 3, Pages 315-352

S 0273-0979(01)00909-0

Article electronically published on March 27, 2001

\title{
A BRIEF HISTORY OF THE CLASSIFICATION OF THE FINITE SIMPLE GROUPS
}

\author{
RONALD SOLOMON
}

\begin{abstract}
We present some highlights of the 110-year project to classify the finite simple groups.
\end{abstract}

\section{The Beginnings}

"Es wäre von dem grössten Interesse, wenn eine Uebersicht der sämmtlichen einfachen Gruppen von einer endlichen Zahl von Operationen gegeben werden könnte." ["It would be of the greatest interest if it were possible to give an overview of the entire collection of finite simple groups."] So begins an article by Otto Hölder in Mathematische Annalen in $1892[\mathrm{Ho}$. Insofar as it is possible to give the birthyear of the program to classify the finite simple groups, this would be it. The first paper classifying an infinite family of finite simple groups, starting from a hypothesis on the structure of certain proper subgroups, was published by Burnside in 1899 [Bu2]. As the final paper (the classification of quasithin simple groups of even characteristic by Aschbacher and S. D. Smith) in the first proof of the Classification Theorem for the Finite Simple Groups (henceforth to be called simply the Classification) will probably be published in the year 2001 or 2002, the classification endeavor comes very close to spanning precisely the 20th century.

Of course there were some important pre-natal events. Galois introduced the concept of a normal subgroup in 1832, and Camille Jordan in the preface to his Traité des substitutions et des équations algebriques in 1870 [J1] flagged Galois' distinction between groupes simples and groupes composées as the most important dichotomy in the theory of permutation groups. Moreover, in the Traité, Jordan began to build a database of finite simple groups - the alternating groups of degree at least 5 and most of the classical projective linear groups over fields of prime cardinality. Finally, in 1872, Ludwig Sylow published his famous theorems on subgroups of prime power order $\mathrm{Sy}$.

Nevertheless Hölder's paper is a landmark. Hölder threw down a gauntlet which was rapidly taken up by Frank Cole, who in 1892 [Co1] determined all simple groups of orders up to 500 (except for some uncertainties related to 360 and 432) and in 1893 Co2 extended this up to 660, discovering in the process a new simple group $S L(2,8)$. By the dawn of the 20th century Miller and Ling (1900) [ML] had pushed this frontier out to 2001. These results were achieved with the only available tools - Sylow's Theorems and the Pigeonhole Principle. Needless to say, the arsenal of

Received by the editors September 18, 2000, and in revised form December 15, 2000.

2000 Mathematics Subject Classification. Primary 20D05.

Research partially supported by an NSF grant. 
weapons needed to be enlarged and the strategy of proceeding one integer at a time needed to be abandoned if any serious progress was to be made.

An alternate strategy was already implicit in a lemma of Hölder (1892) [Ho] proving that a simple group whose order is a product of at most three prime numbers must be cyclic of prime order. The idea that the structure of a finite group $G$ depends more on the shape of the prime factorization of $|G|$ than on the actual nature of the prime factors was explicitly stated by Cole and Glover (1893) [CG] in their critique of Cayley and Kempe: "It is however a defect of their method of classification that it proceeds simply according to the order and not the type of the groups. Thus the groups of order $p q$ where $p$ and $q$ are prime numbers are all of one of two types, the orders $10,14,15, \ldots$ presenting no greater complexity than the order 6." This vein was explored further by Burnside and Frobenius, who both established by 1895 that the only nonabelian simple groups whose order is a product of at most five prime factors are $\operatorname{PSL}(2, p)$ for $p \in\{5,7,11,13\}$. As remarked by Peter Neumann, it is an open question analogous to the Twin Primes Conjecture whether there are infinitely many primes $p$ for which $|P S L(2, p)|$ is a product of exactly six prime factors. Thus again this strategy rapidly encounters difficult and irrelevant obstacles. Its last hurrah was Burnside's proof in 1900 Bu3] that if $G$ is a nonabelian simple group of odd order, then $|G|$ must be a product of at least seven prime numbers.

In a similar but more fruitful direction, Frobenius [Fr1] proved in 1893 that a simple group of squarefree order must be cyclic of prime order. Burnside extended this in 1895 [Bu1] to the following suggestive result:

Theorem. If $p$ is the smallest prime divisor of $|G|$ and if $G$ has a cyclic Sylow p-subgroup $P$, then $G=K P$, where $K$ is a normal subgroup of order prime to $p$. In particular if $G$ is simple, then $|G|=p$.

This points to the importance of the smallest prime divisor $p$ of $|G|$ and to the significance of the structure of a Sylow $p$-subgroup, not simply its cardinality.

Thus by 1895 the strategies were becoming more subtle, but the techniques had not advanced beyond Sylow and virtuoso counting arguments. All of this began to change on April 6, 1896, when Dedekind wrote his famous letter to Frobenius inviting him to consider the problem of factoring the group determinant of a finite nonabelian group. This problem, which vastly generalizes the problem of factoring the determinant of circulant matrices, is roughly equivalent to the decomposition of the regular representation of a finite group into its irreducible constituents, or the complex group algebra as a sum of simple two-sided ideals. The solution by Frobenius later that year signalled the birth of the theory of group characters, which he rapidly developed to the point where he could give a recursive algorithm for the computation of the group characters of the symmetric groups $S_{n}$ for all $n$. Nevertheless it was not immediately clear whether this new theory of group characters was of any use for the structure theory of finite groups.

This question was resolved in the affirmative by Burnside in $1900 \mathrm{Bu} 3$, when he used the new theory to prove that a transitive permutation group of prime degree $p$ either must be 2-transitive or must have a normal Sylow $p$-subgroup of order $p$. Since a 2-transitive group $G$ of degree $p$ must have $|G|$ divisible by $p(p-1), G$ must in particular either be of even order or be solvable. Using this, Burnside was able to show that if $G$ is a nonabelian simple group of odd order, then $|G|>40000,|G|$ must have at least seven prime factors, and $G$ can have no proper subgroup of index 
less than 101. This prompted his famous observation in 1911 [Bu5]: "The contrast that these results shew between groups of odd and even order suggests inevitably that [nonabelian] simple groups of odd order do not exist."

The less-than-friendly rivalry between Frobenius and Burnside produced two further spectacular applications of group characters. Both represented the culmination of a sequence of partial results by both researchers. First came Frobenius' Theorem. We call a permutation group $G$ on a set $X$ regular if, for any two points $x, y \in X$, there exists a unique $g \in G$ with $g(x)=y$. Every group acts regularly on itself via the regular representation. Thus regular action puts no restriction on the structure of $G$. On the other hand Frobenius [Fr2] proved the following result.

Frobenius' Theorem. Let $G$ be a permutation group which acts transitively but not regularly on a finite set $X$. Suppose that no nonidentity permutation in $G$ fixes more than one point. Then $G=K G_{x}$, where $K$ is a regular normal subgroup of $G$ and $G_{x}$ is the stabilizer of the point $x$. In particular $G$ is not a simple group.

There is an equivalent theorem in the context of abstract groups. If $H$ is a subgroup of $G$, we let $H^{g}=g^{-1} H g$.

Theorem. Let $G$ be a finite group with a proper subgroup $H$ such that $H \cap H^{g}=1$ for all $g \in G-H$. Then $G$ has a proper normal subgroup $K$ such that $G=K H$ and $K \cap H=1$.

Groups $G$ satisfying the hypotheses of either version of Frobenius' Theorem are called Frobenius groups, and the subgroups $H$ and $K$ are called a Frobenius complement (unique only up to $G$-conjugacy) and the Frobenius kernel respectively. In the proof Frobenius used his Reciprocity Theorem. As clarified later by Brauer and Suzuki, the key is the decomposition of certain virtual characters induced from $H$ to $G$, yielding characters of $G$ which restrict irreducibly to $H$. Then $K$ may be recognized as the kernel of a suitable character of $G$.

The final triumph of this era of the Classification was Burnside's proof in 1904 [Bu4] of the $p^{a} q^{b}$ Theorem, using the arithmetic of group characters.

Burnside's $p^{a} q^{b}$ Theorem. Let $G$ be a finite group such that $|G|=p^{a} q^{b}$ for some primes $p$ and $q$. Then $G$ is a solvable group.

Burnside had great hopes for further applications of character theory to the study of finite groups, in particular to the proof of the nonexistence of nonabelian simple groups of odd order. This would have to wait half a century. But before moving on, it is worthwhile to mention the early developments in the American school.

A vigorous school of mathematics was nurtured at the University of Chicago in the 1890's by E.H. Moore, and finite group theory was one of its major interests. Moore proved that any complex linear representation of a finite group is a unitary representation, and using this Maschke proved that every complex linear representation is completely reducible. Moore's student, L.E. Dickson, extended Jordan's database of simple groups to include all the classical projective groups over finite fields in his book Linear Groups (1900) [D1]. Dickson was well aware of the analogy between his work and the recent monumental results of Killing and Cartan classifying the simple continuous groups of Lie, and shortly thereafter $(1901,1903)$ [D2, [D3] he succeeded in constructing analogues of the Lie group $G_{2}$ over finite fields $F$ and establishing their simplicity when $|F|>2$. He also studied finite analogues of $E_{6}$ but did not prove their simplicity. 


\section{THEORY BUILDING}

Dickson declared finite group theory to be dead in the 1920's. Indeed there was something of a hiatus from World War I to the 1930's. Nevertheless there were soon important new developments. First came the work of Philip Hall, Burnside's intellectual heir. Hall acknowledged this genealogy in 1942 at the time of his election to the Royal Society (quoted in [R] $]$ ):

"The aim of my researches has been to a very considerable extent that of extending and completing in certain directions the work of Burnside. I asked Burnside's advice on topics in group theory which would be worth investigation and received a postcard in reply containing valuable suggestions as to worthwhile problems. This was in 1927 and shortly afterwards Burnside died. I never met him, but he has been the greatest influence on my ways of thinking."

Hall undertook in 1932 [H2] "the first stages of an attempt to construct a systematic general theory of groups of prime-power order," justifying this project with the sentence: "It is widely recognised, I believe, that the astonishing multiplicity and variety of these groups is one of the main difficulties which beset the advance of finite-group-theory." Indeed one feature of the later history of the Classification is an assiduous and largely successful effort to avoid having to study any $p$-groups beyond those masterfully analyzed by Philip Hall.

Even more important for the future theory of simple groups was the series of papers Hall published in 1928 [H1] and 1937 [H3], [H4] on finite solvable groups. In the first he establishes generalizations of Sylow's Theorems for finite solvable groups; namely if $G$ is a finite solvable group of order $m n$ with $m$ and $n$ coprime, then $G$ possesses a subgroup $H$ of order $m$, any two such subgroups are conjugate and any subgroup of order dividing $m$ is contained in a conjugate of $H$. Such a subgroup $H$ is now called a Hall subgroup or Hall $\pi$-subgroup where $\pi$ is the set of prime divisors of $m$. More striking were Hall's results of 1937 showing that the existence of Hall $\pi$-subgroups is a characteristic property of finite solvable groups. Thus, although a nonsolvable group $G$ may have Hall $\pi$-subgroups for certain sets of primes, e.g. $\{2,3\}$ for $A_{5}$ and $P S L(2,7)$, the following theorem holds.

P. Hall's Theorem. $G$ is a finite solvable group if and only if, for each expression $|G|=m n$ with $m$ prime to $n, G$ contains at least one subgroup of order $m$ and at least one subgroup of order $n$.

This theorem extends Burnside's $p^{a} q^{b}$ Theorem in a very suggestive fashion, relating the existence of nontrivial normal subgroups in a group $G$ to the factorization of $G$ as a product of permutable subgroups $G=M N=N M$ (of coprime orders). The connection between solvability and factorizations was explored further in the 1950's by Wielandt, Hall, Kegel and others. Finally this line of thought culminated in the factorization theorems of Thompson, which are among the key ideas of the classification proof. Hall's elegant and elementary proof relies on Burnside's theorem as the base case. Coming full circle, Thompson's factorization theorems and related ideas of Bender would finally lead in 1973 to a character-free proof of Burnside's Theorem by Goldschmidt [Go1] and Matsuyama [Ma].

In Germany the 1920's witnessed intense activity on the structure theory of many algebraic objects - fields, division algebras, rings with ACC or DCC, etc. Interest in finite groups led to important papers in the late 1930's by Fitting, Wielandt and 
Zassenhaus in particular. The climate of thought is so well captured by Zassenhaus in the preface to his 1937 book Lehrbuch der Gruppentheorie (translated into English and published by Chelsea in 1949 [Z3]) that I quote it at length:

"Investigations published within the last fifteen years have greatly deepened our knowledge of groups and have given wide scope to group-theoretic methods. As a result, what were isolated and separate insights before, now begin to fit into a unified, if not yet final, pattern....

"It was a course of E. Artin, given in Hamburg during the Winter Semester of 1933 and the Spring Semester of 1934, which started me on an intensive study of group theory. In this course, the problems of the theory of finite groups were transformed into problems of general mathematical interest. While any question concerning a single object (e.g., finite group) may be answered in a finite number of steps, it is the goal of research to divide the infinity of objects under investigation into classes of types with similar structure.

"The idea of $\mathrm{O}$. Hölder for solving this problem was later made a general principle of investigation in algebra by E. Nöther. We are referring to the consistent application of the concept of homomorphic mapping. With such mappings one views the objects, so to speak, through the wrong end of a telescope. These mappings, applied to finite groups, give rise to the concepts of normal subgroups and of factor groups. Repeated application of the process of diminution yields the composition series, whose factor groups are the finite simple groups. These are, accordingly, the bricks of which every finite group is built. How to build is indicated - in principle at least - by Schreier's extension theory. The Jordan-Hölder-Schreier theorem tells us that the type and the number of bricks is independent of the diminution process. The determination of all finite simple groups is still the main unsolved problem." ("Als ungelöstes Hauptproblem verbleibt die Bestimmung aller endlichen einfachen Gruppen.")

In contrast to earlier texts by Burnside Bu5, Miller, Blichfeldt and Dickson [MBD] (1916) and Speiser [Sp] (1927), Zassenhaus' work almost completely omits the theories of permutation groups and of group characters, focussing singlemindedly on the architectural structure of groups in terms of normal subgroups and factor groups. In addition to his improved treatment of the Jordan-Hölder-Schreier Theorem, the principal additions are an exposition and amplification of the theory of group extensions developed by Schur and Schreier. Most notable in this context is the following theorem.

Schur-Zassenhaus Theorem. Let $G$ be a finite group with a normal subgroup $N$ such that $|N|$ and $|G / N|$ are coprime. Then the extension $G$ splits over $N$; i.e. there is a subgroup $H$ of $G$ with $G=N H$ and $N \cap H=1$. Moreover if either $N$ or $G / N$ is a solvable group, then all complements to $N$ in $G$ are $G$-conjugate.

Zassenhaus notes that the conjugacy of complements had been conjectured to hold without any restriction of solvability and observes that the problem had been reduced by Witt to the case where $N$ is simple and $C_{G}(N)=1$; i.e. $G$ is a subgroup of $\operatorname{Aut}(N)$. He then observes that the desired general theorem would follow from the proof of either of the following conjectures:

Odd Order Conjecture (Miller, Burnside). Every finite group of odd order is solvable. 
Schreier Conjecture. If $N$ is a nonabelian finite simple group, then Aut $(N) / N$ is a solvable group.

Both conjectures turned out to be true, but very deep. Indeed the only known proof of the Schreier Conjecture is as a corollary of the Classification Theorem. The proof of the Odd Order Conjecture by Feit and Thompson [FT finally yielded the unrestricted Schur-Zassenhaus Theorem, for which there is still no known elementary proof.

In the same architectural spirit was the paper "Beiträge zur Theorie der endlichen Gruppen" by Fitting [F], edited and published posthumously by Zassenhaus in 1938 . In this paper Fitting took a somewhat different approach to the group extension problem and most notably focussed attention on what came to be called the Fitting subgroup $F(G)$ of a finite group, $G$, namely the join of all normal nilpotent subgroups of $G$. He showed that $F(G)$ is itself nilpotent, hence the unique largest normal nilpotent subgroup of $G$. Moreover for solvable groups $G$ he singled out the following fundamental property.

Fitting's Theorem. Let $G$ be a finite solvable group. Then $C_{G}(F(G)) \leq F(G)$.

Since $F(G)$ is a normal (indeed, characteristic) subgroup of $G$, the conjugation action of $G$ on the elements of $F(G)$ defines a homomorphism of $G$ into $\operatorname{Aut}(F(G)$ ) whose kernel is $C_{G}(F(G))$. Thus an equivalent formulation of Fitting's Theorem is the following result.

Theorem. Let $G$ be a finite solvable group. Then $G / Z(F(G))$ is isomorphic to a subgroup of $\operatorname{Aut}(F(G))$.

The corresponding assertion is emphatically false for general finite groups. Indeed if $G$ is a nonabelian simple group, then $F(G)=1$. The search for a good substitute for $F(G)$ when $G$ is non-solvable would occupy the attention of Gorenstein, Walter and Bender in the late 1960's, but even before this, Fitting's Theorem would play an important role in the thinking of Philip Hall in the 1950's.

There were numerous other notable developments in the 1930's. Grün [Gu and Wielandt Wi] refined and extended Burnside's transfer homomorphism. Given a Sylow $p$-subgroup $P$ of a finite group $G$, the idea of the transfer is to use the natural surjection $P \rightarrow P /[P, P]$ to define a homomorphism $V_{G \rightarrow P /[P, P]}$ which under suitable hypotheses may be shown to be nontrivial. The goal is to detect a normal subgroup $N$ of $G$ such that the quotient group $G / N$ is an abelian $p$-group. For example if $G$ is a finite group of order $n$ with a cyclic Sylow 2-subgroup generated by the element $t$, then the regular representation of $G$ represents $t$ as an odd permutation on $n$ letters. In other words the kernel $N$ of the induced homomorphism $G \rightarrow S_{n} / A_{n}$ is a normal subgroup of $G$ with $G / N$ cyclic of order 2. This argument was known to Frobenius and Burnside, and Burnside realized that it could be extended to produce cyclic quotients of order greater than 2 by replacing permutation representations by monomial representations, i.e. by considering homomorphism $G \rightarrow G L(m, \mathbf{C}) / S L(m, \mathbf{C})$. This was refined around the 1930's into the modern definition of the transfer homomorphism, which served as a tool in abelian class field theory as well as finite group theory.

This is a good moment to digress slightly to explain the concept of local group theory which began to emerge at this point. As defined later by Alperin, a local (or $p$-local) subgroup of a finite group $G$ is the normalizer in $G$ of a nonidentity $p$ subgroup of $G$ for some prime $p$. If $G$ is a nonabelian simple group, then every local 
subgroup $N$ is a proper subgroup of $G$. Indeed the local subgroups afford the largest proper subgroups of $G$ whose existence can be predicted a priori from knowledge of $|G|$ alone. If $|G|=p^{a} g_{0}$ with $\left(p, g_{0}\right)=1$, then Sylow's theorems guarantee the existence of subgroups of order $p^{b}$ for $1 \leq b \leq a$ and the normalizers of these subgroups are the local subgroups of $G$. The main challenge confronted in the Classification Project was: Can we use the "global" hypothesis of the simplicity of $G$ to limit (severely) the possibilities for the $p$-local data of $G$, i.e. the isomorphism types and embeddings of the $p$-local subgroups of $G$ ? Or contrapositively, can we show that most sets of hypothetical $p$-local data for $G$ are incompatible with the simplicity of $G$ ? If this were not possible, then any inductive classification enterprise would have necessarily foundered on the shoals of the unthinkably large number of possible sets of $p$-local data.

For example, in the context of the transfer homomorphism, wishful thinking might suggest the following claim: If $G$ has no nontrivial abelian $p$-quotient, then some $p$-local subgroup $N$ of $G$ has no nontrivial abelian $p$-quotient. Unfortunately this statement is false, as is easily seen by inspection of the alternating group $A_{6}$, which has no nontrivial 2-quotient. On the other hand every 2-local subgroup is isomorphic either to a dihedral group of order 8 or to the symmetric group $S_{4}$, both of which have nontrivial 2-quotients. On the bright side, however, Burnside proved that the claim is true when $G$ has an abelian Sylow $p$-subgroup $P$, taking $N=N_{G}(P)$, the normalizer in $G$ of $P$. Wielandt Wi] extended this considerably (for odd $p$ ) to the case when $P$ is a regular $p$-group in the sense of Philip Hall. In particular this covers the cases when $P$ has exponent $p$ and when $P$ has nilpotence class less than $p$ (i.e. the $p$-fold commutator $\left[x_{1}, x_{2}, \ldots, x_{p}\right]=1$ for all $x_{i} \in P$ ).

This is about as far as you can go while focussing on $N_{G}(P)$. The search for suitable alternative $p$-local subgroups led Grün and Wielandt to the concept of a weakly closed subgroup, which would later loom large in the thinking of Thompson.

Definition. Let $H$ be a subgroup of the group $G$. A subgroup $W$ of $H$ is weakly closed in $H$ (with respect to $G$ ) if $W^{g} \leq H$ implies $W^{g}=W$ for all $g \in G$; i.e. $W$ is the unique member of its $G$-conjugacy class which is contained in $H$.

Grün $\mathrm{Gu}$ proved that if the center $Z(P)$ is a weakly closed subgroup of $P$, then $G$ has an abelian $p$-quotient if and only if $N_{G}(Z(P))$ does. This turned out to be extendible, in a spirit similar to Wielandt's extension of Burnside's theorem, to what might be dubbed regularly embedded weakly closed subgroups of $P$. The best result in this vein was achieved in the 1970's by Yoshida [Y].

The most general theorem, again of later vintage, is Alperin's Fusion Theorem (1967) Al], which implies that the existence of abelian $p$-quotients is always determined $p$-locally, i.e. by examination of the full set of $p$-local data for $G$. Indeed it provides much sharper information. Also in the late 1960's, Glauberman G13] defined nontrivial subgroups $K_{\infty}(P)$ and $K^{\infty}(P)$ such that the normalizer of either of them detects nontrivial $p$-quotients of $G$ whenever $p \geq 5$. Ironically, however, by this point the role of the transfer homomorphism in the Classification was rapidly waning. Moreover, as attention focussed, after the Odd Order Theorem, on the 2local structure of $G$, what little interest transfer held focussed mostly on the prime 2, and here the most effective tool proved to be the Thompson Transfer Lemma, which is merely an elementary refinement of the old permutation group argument of Frobenius and Burnside. 
Yet another major development in the 1930's was Zassenhaus' extension of the work of Jordan and Frobenius on transitive permutation groups. Jordan had classified all sharply $k$-transitive permutation groups for $k=2$ and for $k \geq 4$ [J2]. Sharply 2-transitive permutation groups of a finite set $\Omega$ satisfy the hypotheses of Frobenius' Theorem and thus have the structure $G=K G_{\alpha}$ where $K$ is a regular normal subgroup of $G$. As $K-\{1\}$ is permutation-isomorphic to $\Omega-\{\alpha\}$ as $G_{\alpha^{-}}$ set, $K$ must be an elementary abelian $p$-group for some prime $p$ with $|\Omega|=p^{n}$, $n \geq 1$. Burnside attempted a description of the structure of $G_{\alpha}$, but the first correct treatment was given by Zassenhaus in 1936 [Z2]. At the same time, Zassenhaus classified all finite sharply 3-transitive permutation groups $G$ [Z1]. In such a group $G$, the point stabilizer $G_{\alpha}$ is a sharply 2-transitive group. Zassenhaus initiated the investigation of the larger class of finite 2-transitive permutation groups in which a point stabilizer is a Frobenius group. Such groups came to be known as Zassenhaus groups, and their classification in the late 1950's and early 1960's was a major project involving the work of Thompson, Feit, Ito, G. Higman and Suzuki. The most dramatic moment was Suzuki's discovery [Su3] of a new infinite family of finite nonabelian simple Zassenhaus groups, all of order prime to 3 . These groups came to be known as the Suzuki groups, $S z\left(2^{2 n+1}\right), n \geq 1$. Suzuki's work culminated in 1963 with the following theorem [Su4], Su5] which extends his classification of Zassenhaus groups of odd degree.

Suzuki's Theorem. Let $G$ be a finite simple 2-transitive permutation group on a set $\Omega$ with $|\Omega|$ odd. Suppose that for $\alpha, \beta \in \Omega,\left|G_{\alpha \beta}\right|$ is odd and $G_{\alpha \beta}$ has a normal complement $Q$ in $G_{\alpha}$ such that $Q$ is regular on $\Omega-\{\alpha\}$. Then $G \cong P S L\left(2,2^{n}\right)$, $S z\left(2^{2 n+1}\right)$ or $P S U\left(3,2^{n}\right)$.

Probably the deepest group-theoretic work in the 1930's was the investigation of the modular representations of finite groups, primarily by Richard Brauer, who had left Germany in 1933 and settled in Toronto in 1935 after a year as Weyl's assistant at the IAS. In Brauer's modular theory, the local/global principle reaches a higher level of refinement, as Brauer moves back and forth both between the characteristic 0 and the characteristic $p$ representations of a group $G$ and also between the representations of $G$ and the representations of the $p$-local subgroups of $G$. His early work culminated in his paper on the $p$-modular representations of finite groups $G$ such that $|G|$ is divisible by $p$ but not by $p^{2}$, presented to the A.M.S. in 1939 [Br1]. As reported by Feit [Fe, one of Brauer's "motives in studying modular representations was the hope of characterizing certain classical groups over finite fields." Indeed in Br1] he notes applications such as the uniqueness of simple groups of order 5616 and 6048 . The fundamental principles of his analysis later evolved into his general Main Theorems. The calculation of so-called decomposition matrices, which underlie the detailed results he achieved for Sylow groups of prime order, soon founders however on the rocks of wild representation type. Nevertheless Brauer was later able to push through the analysis for certain Sylow 2-subgroups of 2-rank 2, which proved to be just what was needed to obtain group order formulas for simple groups with dihedral, semidihedral and "wreathed" Sylow 2-subgroups. Alas in general his theory was of much less applicability to the Classification than he had hoped. 


\section{The Classification Begins in EARnest}

After the trauma of World War II, the simple group problem recaptured the attention of leading algebraists. "Das interessanteste Problem scheint mir die Aufsuchung aller endlichen einfachen Gruppen zu sein." ["The most interesting problem seems to me to be the classification of all finite simple groups."] So spoke Zassenhaus in his Antrittsvorlesung at the University of Hamburg in 1947 (quoted in [Be5]). The interest in the problem was clear. The best strategy for its solution was not at all clear. Inspired by the work of Killing and E. Cartan, Zassenhaus hoped to linearize the problem by identifying all simple groups as groups of automorphisms of some linear structure, perhaps a finite Lie algebra. This approach gained credibility when Chevalley $\mathrm{Ch}$ found a uniform method to construct finite analogues of the simple complex Lie groups. Supplemented with variations by Steinberg [St and Ree [Re1, [Re2], this furnished a Lie-theoretic context for all of the known finite simple groups except for the alternating groups and the five Mathieu groups. However, although this yielded a rich harvest of new finite simple groups and a unified context for the study of their subgroups and representations, it did not immediately suggest a strategy for their classification. Indeed, no one has yet found an a priori method of associating to a finite simple group $G$ an algebra of the "correct" dimension, roughly $\log (|G|)$.

Meanwhile around 1950 several mathematicians independently began parallel investigations which were to prove more immediately fruitful for the classification endeavor. The groups $\operatorname{PSL}(2, q)$ are the most elementary of the nonabelian simple groups, and their subgroups have been well-understood since the time of E.H. Moore and Dickson. When $q$ is a power of 2 , the centralizer of every nonidentity element is abelian and the group is partitioned as a union of abelian (Sylow 2-)subgroups of order $q$ and cyclic (Hall) subgroups of order $q-1$ and $q+1$, any two intersecting only in the identity. When $q \equiv \epsilon(\bmod 4)$ (with $\epsilon= \pm 1)$, the centralizer of every element of order greater than 2 is abelian and the group is partitioned as a union of abelian (Sylow $p$-)subgroups of order $q$ and cyclic subgroups of order $q-\epsilon$ and $\frac{q+\epsilon}{2}$, again any two intersecting only in the identity. On the other hand, if $G$ is a finite group in which the centralizer of every non-identity element is abelian, then it is not hard to see that the commuting graph of nonidentity elements of $G$ is a union of disjoint cliques, corresponding to a partition of $G$ as a union of maximal abelian subgroups. Furthermore the normalizer of any such maximal abelian subgroup $A$ is a Frobenius group with Frobenius kernel $A$. When a group $G$ has such a subgroup structure, the situation is especially favorable for the analysis of induced virtual characters (first studied by Brauer) in the spirit of Frobenius' Theorem. This seems to have been recognized independently around 1950 by Brauer, Suzuki and G.E. Wall, who began parallel investigations.

In 1948 Brauer accepted a chair at the University of Michigan and shortly thereafter, together with his student K.A. Fowler, began the investigation of CA-groups of even order, i.e. groups of even order in which the centralizer of every nonidentity element is abelian. Around the same time, Wall in Manchester began similar research at the suggestion of Graham Higman. (There were some antecedents in the work of Szekeres (1949) [Sz] and Redei (1950) $[\mathrm{Rd}]$.) Meanwhile Suzuki in Japan discovered a characterization of $P G L(2, q), q$ odd, in terms of partitions [Su1], which attracted the attention of Baer, who invited Suzuki to join him at the University of Illinois in 1951. The following summer Suzuki participated in Brauer's summer 
seminar in Michigan, which was also attended by graduate students including Walter Feit and John H. Walter. By 1953, Brauer, Suzuki and Wall had each arrived at characterization theorems for $\operatorname{PSL}(2, q)$ beginning from local data, one version of which would be published several years later as the Brauer-Suzuki-Wall Theorem [BSW]. Surprisingly, the easy case of this theorem together with some of the key non-character-theoretic ideas for its proof had been discovered and published in 1899 by Burnside [Bu2], a result of which deserves recognition as the first classification theorem for finite simple groups:

Theorem (Burnside, 1899). Let $G$ be a finite nonabelian simple group of even order. Suppose that every element of $G$ is either an involution (i.e. an element of order 2 ) or is of odd order. Then $G \cong S L\left(2,2^{n}\right)$ for some integer $n \geq 2$.

Note that Burnside's hypothesis can be reformulated in the CA-spirit as follows:

Suppose that $G$ is a group of even order in which the centralizer of every involution of $G$ is an abelian group of exponent 2 .

Burnside's beautiful paper dwelt in undeserved obscurity until it was rediscovered by Walter Feit around 1970 to the great surprise of Brauer. It is an example of an important paper which had no impact on the history of the field.

In 1952 Brauer moved to Harvard and in 1954 he addressed the International Congress of Mathematicians, beginning with the following words:

"The theory of groups of finite order has been rather in a state of stagnation in recent years. This has certainly not been due to a lack of unsolved problems. As in the theory of numbers, it is easier to ask questions in the theory of groups than to answer them. If I present here some investigations on groups of finite order, it is with the hope of raising new interest in the field."

Brauer focussed primarily on groups of even order and announced, in addition to the Brauer-Suzuki-Wall Theorem on $\operatorname{PSL}(2, q)$, the Brauer-Fowler bound on the order of a finite simple group of even order, given the order of one of its involution centralizers, and a characterization of $\operatorname{PSL}(3, q)$ and $M_{11}$ via the centralizer of an involution. Because of its generality, the Brauer-Fowler Theorem $[\mathrm{BF}]$ had a particularly great psychological impact.

Brauer-Fowler Theorem. Let $G$ be a finite simple group of even order containing an involution $t$. If $\left|C_{G}(t)\right|=c$, then $|G| \leq\left(c^{2}\right)$ !.

The specific bound is useless in practice and even the Brauer-Fowler argument is only of value in a few small cases. Nevertheless the theorem asserts that for any finite group $H$ the determination of all finite simple groups with an involution centralizer isomorphic to $H$ is a finite problem. In the ensuing decades Brauer, Janko and their students would show that the problem was not only finite but tractable. This suggested a two-step strategy for the proof of the Classification Theorem:

Step 1: Determine all possible structures for an involution centralizer in a finite simple group.

Step 2: For each possible structure, determine all finite simple groups with such an involution centralizer.

Brauer had proved some sample cases for Step 2. No one had a clue how to do Step 1. Indeed no one had a clue how to show that a nonabelian finite simple group even contains an involution. Or did they? Just a few months after the Congress, on December 24, 1954, a historic paper was submitted by Suzuki Su2 
to the Bulletin of the A.M.S. It contained a beautiful proof of the nonexistence of nonabelian simple CA-groups of odd order. This was the first breakthrough in the direction of the Miller-Burnside conjecture concerning the solvability of groups of odd order. Suzuki's CA-group proof had two parts. As mentioned above, a rather easy "local" analysis gives the structure of the maximal subgroups of a CA-group G. Then a brilliant application of exceptional character theory leads to a contradiction. Thompson writes [T4]:

"Suzuki's CA-theorem is a marvel of cunning.... Once one accepts this theorem as a step in a general proof, one seems irresistibly drawn along the path which was followed [in the Odd Order Paper]."

Nevertheless the difficulties for the proof of the Miller-Burnside Odd Order Conjecture still seem insuperable. In Suzuki's case, the maximal subgroups of $G$ are easily seen to be Frobenius groups. In the case of an arbitrary finite simple group of odd order, one could assume by induction that every maximal subgroup was a solvable group. But the possible structures of solvable groups are far more elaborate than the structures of Frobenius groups. Without major reductions using local analysis, the requisite character theory would be unthinkably difficult.

The next breakthrough came from an unexpected direction. There was considerable activity in the 1950's on the Burnside and Restricted Burnside Problems, including Kostrikin's proof of the Restricted Burnside Theorem for groups of prime exponent. In this context Philip Hall and Graham Higman wrote a remarkable paper $[\mathrm{HH}]$ in 1956 aimed at the reduction of the Restricted Burnside Problem to the prime-power exponent case. They provided an insightful analysis of the structure of finite $p$-solvable groups (a class containing all solvable groups). In particular they established the following results.

Hall-Higman Lemma. Let $G$ be a finite $p$-solvable group and let $X$ be the largest normal subgroup of order prime to $p$. Let $P$ be a p-subgroup of $G$ such that $X P / X$ is the largest normal p-subgroup of $G / X$ and let $V=P / \Phi(P)$. Then $H=G / X P$ acts by conjugation as a faithful p-solvable group of linear operators on the vector space $V$ and $H$ has no nontrivial normal p-subgroup.

Hall-Higman Theorem B. Let $H$ be a p-solvable group of linear operators on a finite-dimensional vector space $V$ over a field of characteristic $p$. Suppose that $H$ has no nontrivial normal $p$-subgroup. If $x \in H$ with $x^{p}=1 \neq x$, then either the minimum polynomial of $x$ in its action on $V$ is $(t-1)^{p}$ or the following conclusions hold: $p$ is a Fermat prime, $H$ has a nonabelian Sylow 2-subgroup and the minimum polynomial of $x$ is $(t-1)^{p-1}$.

The Hall-Higman theorems conveyed some important messages. First of all, if $G$ is a complicated finite solvable group (in particular, far from nilpotent), then $G$ has a comparatively uncomplicated normal subgroup $N=O_{p^{\prime} p}(G)$ with the property that $G / N$ is isomorphic to a group of linear operators on a vector space $V$ arising as a quotient of $N$. Secondly, Theorem B shows how linear algebra can be exploited to analyze the structure of $G / N$ and it displays a distinction between the structure of $p$-solvable groups and the structure of many parabolic subgroups in groups of Lie type in characteristic $p$. For example, if $H$ is the stabilizer of a 1-space in $G L(n+1, p), N=O_{p^{\prime}}(H)$ and $Z=Z(H)=O_{p^{\prime}}(H)$, then $N=Z \times V$, where $V=O_{p}(H)$ which may be thought of as an $n$-dimensional vector space over $\mathbf{F}_{p}$. Moreover $\bar{H}=H / N$ is isomorphic to $G L(n, p)$ acting naturally on $V$. Thus 
$\bar{H}$ has no nontrivial normal $p$-subgroup, but if $p$ is odd, then for many $x \in \bar{H}$ of order $p$, the minimum polynomial of $x$ in its action on $V$ has degree less than $p$. Indeed the transvections have quadratic minimum polynomial $(t-1)^{2}$. Of course $\bar{H}$ is not a $p$-solvable group when $p>3$. (When $p=3$, we are precisely in one of the exceptional cases of Theorem B: $H$ has a normal quaternion subgroup and 3 is a Fermat prime.)

Thus far the work of the 1950's could be regarded as the brilliant belated fulfillment of the work of the period 1890-1910. Indeed Chevalley and Steinberg had created the analogue over finite fields of the Lie theory of Killing and Cartan, completing the work of Dickson. Brauer, Suzuki and Feit had extended the character theory of Frobenius and Burnside and its applications to the classification of small simple groups. The study of Zassenhaus groups by Feit, Ito and Suzuki was nearing completion, tapping out a vein of permutation group theory going back to Jordan. Some pregnant possibilities lay in the papers of Suzuki and Hall-Higman. Nevertheless there was some truth to Brauer's assertion at the International Congress of Mathematicians in 1970 on the occasion of Thompson's receiving the Fields Medal: "...up to the early 1960's, really nothing of real interest was known about general simple groups of finite order."

\section{Enter John ThOMPson}

At the suggestion of Marshall Hall, Thompson attacked in his dissertation T1] the long-standing conjecture that the Frobenius kernel is always nilpotent. This is equivalent to the following assertion:

Thompson's Thesis. Let $G$ be a finite group admitting an automorphism $\alpha$ of prime order with $C_{G}(\alpha)=1$. Then $G$ is a nilpotent group.

If $G$ is nilpotent, then for every prime $p$ dividing $|G|, G$ has a normal subgroup of index $p$. Thus it is natural to attack the problem via transfer. Choosing an $\alpha$-invariant Sylow $p$-subgroup $P$ of $G$, induction applies to the normalizer of characteristic subgroups of $P$. It follows from Grün's Second Theorem $\mathrm{Gu}$ that $Z(P)$ cannot be weakly closed in $P$. This led Thompson to the study of weak closures of abelian subgroups of $P$.

Definition. Let $A \leq H \leq G$. The weak closure of $A$ in $H$ with respect to $G$ is

$$
W=\left\langle A^{g}: A^{g} \leq H\right\rangle .
$$

Equivalently, $W$ is the smallest subgroup of $H$ containing $A$ and weakly closed in $H$ (with respect to $G$ ).

Thompson's analysis is quite delicate but eventually leads to a Hall-Higmantype situation involving a pair of elementary abelian $p$-groups $A$ and $B$ which normalize each other but do not commute. Thus $[A, B] \neq 1$ but $[A, B] \leq B$ and so $[A, B, B]=1$. Hence some element $x$ of $B$ viewed as a linear operator on $A$ has quadratic minimum polynomial. Thanks to freedom in the choice of $p$, this contradicts the Hall-Higman Theorem B. In fact the quadratic action of $x$ permits a more elementary contradiction, but the shadow of Hall-Higman is definitely visible.

Thompson's thesis had immediate implications for the study of Zassenhaus groups, clarifying as it did the structure of Frobenius kernels. Even more important, it was the beginning of Thompson's profound analysis of the structure 
of solvable subgroups of finite simple groups. In the summer of 1958 while working on his thesis, Thompson visited Wielandt in Tübingen, and Huppert reports Wielandt's comment:

"Das is ein verdammt scharfsinniger Bursche. Man kann etwas lernen von ihm." ["That's one damn sharp guy. You can learn something from him."]

Thompson completed his theorem and sent his work to Philip Hall in December 1958. Hall immediately grasped the import of Thompson's achievement and suggested a reformulation of one of the main theorems which liberated it from the context of groups with operators.

Theorem. Let $K$ be a finite group, $p$ an odd prime and $P$ a Sylow $p$-subgroup of $K$. Suppose that $K \neq X P$ for any normal $p^{\prime}$-subgroup $X$. Then there exists a characteristic subgroup $D$ of $P$ of nilpotence class at most 2 such that $N_{K}(D) / C_{K}(D)$ is not a p-group.

Further consideration of weak closure and the Hall-Higman Theorem B led Thompson to the discovery of the $J$-subgroup and the Thompson factorization theorems. There are two slightly different definitions of the $J$-subgroup. I shall give the one which has become more popular recently.

Definition. Let $P$ be a finite $p$-group and let $d$ be the maximum rank of an elementary abelian subgroup of $P$. Let $\mathcal{A}(P)$ denote the set of all elementary subgroups of $P$ of rank $d$. Then the Thompson subgroup $J(P)$ is

$$
J(P)=\langle A: A \in \mathcal{A}(P)\rangle .
$$

Let $H$ be a finite solvable group whose Fitting subgroup $F$ is a $p$-group. For $R$ any $p$-group denote by $\Omega_{1}(R)$ the subgroup generated by the elements of order $p$ in $R$. If $P$ is a Sylow $p$-subgroup of $H$, then $Z(P) \leq Z(F)$ by Fitting's Theorem and $\mathrm{SO}$

$$
V=V_{H}=\left\langle\Omega_{1}\left(Z\left(P^{h}\right)\right): h \in H\right\rangle
$$

is a subgroup of $\Omega_{1}(Z(F))$. In particular $V$ may be regarded as an $H$-module on which $F$ acts trivially. The subgroup $C$ which is the kernel of the $H$-action on $V$ may be larger than $F$, but it shares with $F$ the property that $H / C$ has no nontrivial normal $p$-subgroup. Thus $H / C$ may be regarded as a solvable subgroup of $G L(V)$ with no nontrivial normal $p$-subgroup, exactly the Hall-Higman setup. Under suitable additional hypotheses, for example that $H$ has odd order, Thompson shows that no $A \in \mathcal{A}(P)$ can act nontrivially on $V$, i.e. $J(P) \leq C$. But then Sylow's Theorem immediately yields the Thompson Factorization:

$$
H=C N_{H}(J(P))=C_{H}\left(\Omega_{1}(Z(P))\right) N_{H}(J(P)) .
$$

When hypotheses such as solvability and odd order are dropped, the analysis becomes much more complicated, but the fundamental philosophy remains the same.

Definition. A finite group $G$ is of (local) characteristic $p$-type if the following condition is satisfied by every $p$-local subgroup $H$ of $G$ : Let $F$ be the largest normal $p$-subgroup of $H$. Then $C_{H}(F) \leq F$.

Whenever $G$ is a group of characteristic $p$-type, Thompson's analysis may be undertaken. Having chosen a Sylow $p$-subgroup $P$ of $G$, it shows that there are two $p$-local subgroups of fundamental importance: $C=C_{G}\left(\Omega_{1}(Z(P))\right)$ and $N=$ 
$N_{G}(J(P))$. If $H=N_{G}(D)$ for some nonidentity normal subgroup $D$ of $P$ and $H \neq$ $(H \cap C)(H \cap N)$, then $V_{H}$ must be a "failure of factorization module" for $H / C_{H}\left(V_{H}\right)$. As stated here, this is a linguistic tautology, but in fact it has mathematical content. In particular, failure of factorization modules are "quadratic modules", and around 1970, Thompson [T3 classified all "quadratic pairs" for $p \geq 5$, i.e. pairs $(H, V)$ where $V$ is a faithful irreducible quadratic module for $H$ in characteristic $p$ and $H$ is generated by its elements of order $p$ having quadratic minimum polynomial. Necessarily $H$ is a product of groups of Lie type in characteristic $p$ and $V$ is a tensor product of small modules. This has been extended to the case $p=3$, where new examples arise such as spin modules for the spin covers of the alternating groups and the Leech lattice mod 3 for the Conway group and some of its subgroups. When $p=2$, all involutions act quadratically, but the analysis of failure of factorization modules remains meaningful and is at the heart of the classification of simple groups of characteristic 2-type.

Glauberman's discovery of the $Z J$-Theorem [G12] around 1967 provided an easier approach than factorization theorems in the context of groups of odd order. Recently Stellmacher S11] has established an analogue of the $Z J$-Theorem for groups of order prime to 3 . However, in the general context, when the primes 2 and 3 are intertwined in the subgroup structure of $G$, Thompson's more robust factorization approach returns to center stage.

By 1959 when Marshall Hall published his text The Theory of Groups [Ha], he could write in dramatic contrast to Brauer's remarks in 1954: "Current research in Group Theory, as witnessed by the publications covered in Mathematical Reviews, is vigorous and extensive." Hall invited Thompson to Caltech in the summer of 1959 and they extended Suzuki's theorem on CA-groups of odd order to the nilpotent centralizer case. They sent a copy of the manuscript to Feit, who substantially improved the character theory. This launched the collaboration of Feit and Thompson on groups of odd order. Suzuki's CA-paper was 10 pages in length. The CN-paper [FHT] was 17 pages. Feit and Thompson estimated it would take about 25 pages to prove the Odd Order Theorem. That proved to be quite an underestimate.

Fundamental to the analysis of the minimal simple group $G$ of odd order as pursued in the Odd Order Paper is the dichotomy between those groups $G$ in which the intersection $M \cap M^{g}$ is always "small", for $M$ a maximal subgroup of $G$ and $g \in G-M$, and those groups $G$ in which the intersection is sometimes "large". The former case was primarily to be handled by extending the Brauer-SuzukiFeit analysis of exceptional characters to an even more complicated setting. The local methods Feit and Thompson introduced to treat the latter case had profound impact on the rest of the Classification. By contrast the Odd Order Paper was the high watermark for character theory in the Classification. Future applications were few and far less intricate. Because of the greater resonance of the local methods in future papers, I shall discuss them along with related generalizations in the remainder of this section.

In their attack on the "large" case, Feit and Thompson were motivated by an important paper of Philip Hall [H5] from 1956, extending his earlier work on criteria for solvability in terms of permutability of Sylow subgroups. Hall introduced the symbol $E_{p q}$ to denote the existence in $G$ of a Hall $\{p, q\}$-subgroup. As Thompson's notes in T4, Hall's 1956 paper "suggests that a group is solvable if and only if it satisfies $E_{p, q}$ for all primes $p, q$." Thompson undertook to prove such $E$-theorems for groups of odd order. He writes: 
"From my work on the Frobenius kernel, I wanted to work upwards from the bottom, and the bottom is the Fitting subgroup. So, in trying to prove $E_{p, q}, \mathrm{I}$ could see the value in trying to locate $q$-subgroups $Q$ which are normalized by a Sylow p-subgroup of $G$."

This led to the fundamental concept of $A$-signalizers.

Definition. Let $A$ and $H$ be subgroups of the group $G$ with $A$ a $p$-group and let $\pi$ be a set of primes not containing $p$. The set $\Lambda_{H}(A ; \pi)$ is the set of all $A$-invariant $\pi$-subgroups of $H$, and $И_{H}^{*}(A ; \pi)$ is the set of maximal elements of $И_{H}(A ; \pi)$ under inclusion.

Thus in this language Thompson was seeking $Q \in И_{G}(P ; q)$ for $P$ a Sylow $p$ subgroup of $G$. If $H$ is an $A$-invariant solvable $p^{\prime}$-group, then by Philip Hall's theorem, the members of $И_{H}^{*}(A ; \pi)$ are Hall $\pi$-subgroups of $H$ and are transitively permuted by $C_{H}(A)$. Thompson saw how to extend this result to the context of local solvability.

Thompson Transitivity Theorem. Let $G$ be a finite group in which every $p$ local subgroup is solvable. Let $A$ be an abelian p-group of rank at least 3 which is a Sylow p-subgroup of $C_{G}(A)$. Then $C_{G}(A)$ transitively permutes the elements of $U_{G}^{*}(A ; q)$ for all primes $q \neq p$.

If $P$ is a Sylow $p$-subgroup of $G$ containing $A$, then as $N_{G}(A)$ permutes the elements of $И_{G}^{*}(A ; q)$ with $C_{G}(A)$ acting transitively, Lagrange's Theorem yields that $N_{P}(A)$ normalizes some $Q \in И_{G}^{*}(A ; q)$. Bootstrapping upward often yields some $Q \in U_{G}^{*}(P ; q)$, as Thompson desired.

The requirement that $A$ have $p$-rank at least 3 is unavoidable and makes precise the above-mentioned subdivision of the analysis into small and large cases, not only in the Odd Order Paper but throughout the Classification. The Transitivity Theorem is a key tool in the proof of the Uniqueness Theorem below, which is the basic result for eliminating the "large" case:

The Feit-Thompson Uniqueness Theorem. Let $G$ be a finite group of odd order in which every proper subgroup is solvable. Suppose that $K$ is a proper subgroup of $G$ such that either $r(K) \geq 3$ or $r\left(C_{G}(K)\right) \geq 3$. Then $K$ is contained in a unique maximal subgroup of $G$. (Here $r(K)$ denotes the maximum rank of an abelian $p$ subgroup of $K$, as $p$ ranges over all prime divisors of $|K|$.)

A beautiful proof of the Uniqueness Theorem, incorporating Glauberman's $Z J$ Theorem and his own new ideas, was discovered by Bender [Be1 in the late 1960's. Around the same time, Gorenstein and Walter undertook a profound analysis of the Feit-Thompson signalizer arguments with the goal of extending the analysis to a context where $p$-local subgroups are no longer solvable. They solved this problem with the concept of an $A$-signalizer functor, which evolved over the years, the following elegant definition being due to Goldschmidt Go2].

Definition. Let $A$ be an abelian $p$-subgroup of the finite group $G$. Then a function $\theta$ mapping the set $A^{\#}$ into the set of solvable $A$-invariant $p^{\prime}$-subgroups of $G$ is called a solvable $A$-signalizer functor if $\theta(a)=\theta\left(C_{G}(a)\right) \leq C_{G}(a)$ for all $a \in A^{\#}$ and the following "balance equation" holds for all $a, b \in A^{\#}$ :

$$
\theta\left(C_{G}(a)\right) \cap C_{G}(b)=\theta\left(C_{G}(b)\right) \cap C_{G}(a) .
$$


They then focussed attention on the sets $И_{\theta}(A, \pi)$ consisting of those members $X$ of $\bigcup_{G}(A, \pi)$ such that $C_{X}(a) \leq \theta\left(C_{G}(a)\right)$ for all $a \in A^{\#}$. Finally $И_{\theta}(A)$ is the union of all of these sets, and the Solvable Signalizer Functor Theorem states:

Solvable Signalizer Functor Theorem. Let $G$ be a finite group, $A$ an abelian p-subgroup of $G$ with $r(A) \geq 3$ and $\theta$ a solvable $A$-signalizer functor. Then $И_{\theta}(A)$ has a unique maximal element $\theta(G)$.

Early versions of this theorem were proved around 1969 by Gorenstein [G2]. The full theorem for $p=2$ was established by Goldschmidt Go3, and then finally the general case was proved by Glauberman [GI4] in 1973. The proof entails remaining in the realm of solvable $\theta$-subgroups of $G$ where it is possible to recover some of Thompson's results, such as the Transitivity Theorem. A Nonsolvable Signalizer Functor Theorem was proved in the late 1970's by McBride [McB1], McB2].

Once $\theta(G)$ exists, the fundamental dichotomy is:

$$
\theta(G) \neq 1 \text { or } \theta(G)=1 \text {. }
$$

When $\theta(G) \neq 1$, the simplicity of $G$ implies that $M=N_{G}(\theta(G))$ is a proper subgroup of $G$ containing the normalizers of many $p$-subgroups of $G$. We call such a subgroup a $p$-uniqueness subgroup. In many contexts, such as the Odd Order Paper, $\theta(G)=1$ implies that $G$ is of characteristic $p$-type and Thompson factorization analysis may be pursued. In the Odd Order Paper the absence of failure of factorization modules again leads to the existence of $p$-uniqueness subgroups. This is an important milestone, but considerable difficult character theory and generatorand-relations arguments are still necessary to complete the proof of the Odd Order Theorem.

Adrian Albert organized a Group Theory Year at the University of Chicago in 1960-61. This was perhaps the most successful mathematical year ever organized. Feit and Thompson completed most of their work on the Odd Order Theorem. Suzuki pursued his research on 2-transitive permutation groups. Gorenstein and Walter began their collaborative study of groups with dihedral Sylow 2-subgroups. The first real signs appeared of the evolving sociology of the classification effort as a team project. (The earlier Brauer-Suzuki-Wall paper was an instance of parallel research arriving at approximately the same point at the same time.) What also became evident was the unprecedented scale of the undertaking. The Odd Order Paper was an unbelievable 255 pages in length, and there was no fat on the manuscript. Many of the later manuscripts would follow this triple digit pattern, culminating in the 731 page Memoirs volume by Gorenstein and Lyons [GL1] and the forthcoming 800+ page Quasithin Paper by Aschbacher and S. D. Smith [AS].

Feit and Thompson published the Odd Order Paper in 1963 [FT]:

The Odd Order Theorem. All finite groups of odd order are solvable.

This short sentence and its long proof were a moment in the evolution of finite group theory analogous to the emergence of fish onto dry land. Nothing like it had happened before; nothing quite like it has happened since. I compare the character theory (Chapters 3 and 5) to Bach's B Minor Mass, the glorious summation of everything which had been achieved by Frobenius, Brauer, Suzuki and Feit himself, on the theme of wresting information about group structure from the arithmetic of induced characters. It is hard to imagine pushing this analysis through in a more complicated setting. Luckily no one ever needed to. By contrast, I compare the 
local analysis (Chapter 4) to Beethoven's Ninth Symphony. Looking back at earlier group theory it declared: "O Freunde, nicht diese Töne!" Looking ahead to the great collaborative effort of the next 40 years whose size and shape it presaged, it declared: "Sondern lasst uns angenehmere anstimmen!"

\section{BACK TO THE PRIME 2}

Once the Odd Order Paper was completed, attention naturally focussed on the prime 2. The work of Brauer, Suzuki and Wall in the 1950's had shown how the existence of involutions could be exploited to characterize simple groups starting from fairly detailed 2-local data. The Odd Order Paper (and Thompson's evolving work [T2] on minimal simple groups of even order) were the only models for arriving at such detailed local data. The dichotomy which had emerged in the Odd Order Paper between groups of $p$-rank at most 2 and those of $p$-rank at least 3 suggested the importance of groups of 2-rank 2 as a separate problem. (A 2-group of rank 1 is either cyclic or quaternion. An old argument (sketched earlier) shows that no simple group of even order except $C_{2}$ has cyclic Sylow 2-subgroups. Brauer and Suzuki [BS] had proved that no simple group has quaternion Sylow 2-subgroups.) Gorenstein and Walter GW1] completed the dihedral case. Luckily, an elegant argument of Alperin showed that a 2-group of 2-rank 2 which was a candidate to be a Sylow 2-subgroup of a simple group must fall into one of four infinite families (dihedral, semidihedral, wreathed, homocyclic abelian) or be of one exceptional isomorphism type. Alperin's proof was made possible by an elegant application of modular character theory to 2-fusion analysis by Glauberman [G11]:

Glauberman's $Z^{*}$-Theorem. Let $G$ be a finite group with no nontrivial normal subgroup of odd order. Let $z$ be an involution of $G$. Either $z \in Z(G)$ or $z$ commutes with a $G$-conjugate $z^{g}$ with $z^{g} \neq z$.

Alperin and Gorenstein undertook the semidihedral/wreathed case, which turned out to be quite difficult and to require considerable 2-modular character theory, for which they turned to Brauer. By 1969 simple groups of 2-rank at most 2 had been classified [ABG1], ABG2]. Once it could be assumed that $G$ had 2-rank at least 3 , it was at least possible to begin 2-signalizer functor analysis. This was not, however, quite enough to pass gracefully from the conclusion of the Signalizer Functor Theorem for $p=2$ to the existence of a 2-Uniqueness Subgroup of the desired type when $\theta(G) \neq 1$. To bridge this gap Gorenstein and Harada produced a monumental work [GH] classifying simple groups of sectional 2-rank at most 4 . Later Harada [Hr1] discovered a short and elegant argument to build the same bridge, though their magnum opus was quoted in many other contexts.

When a 2-uniqueness subgroup $M$ exists, there remains the problem of identifying the group $G$. The obvious examples are the groups $S L\left(2,2^{n}\right), P S U\left(3,2^{n}\right)$ and $S z\left(2^{n}\right)$, which arise as the conclusions of Suzuki's Theorem on 2-transitive permutation groups. The strongest form of 2 -uniqueness subgroup can be defined in permutation group language as:

Definition. A group $G$ has a strongly embedded subgroup $M$ if $G$ is a transitive permutation group of even order in which every involution fixes exactly one point. ( $M$ is the stabilizer of a point.) 
One obvious gap between this definition and Suzuki's hypotheses is that $G$ is not assumed to be 2-transitive. Indeed this need not be the case if $G$ has 2-rank 1 . However, Bender [Be3] succeeded in 1968 in proving the following beautiful theorem:

Bender's Strongly Embedded Theorem. Let $G$ be a finite simple group with a strongly embedded subgroup $M$. Then $G$ satisfies the hypotheses of Suzuki's Theorem. Thus $G \cong S L\left(2,2^{n}\right), S z\left(2^{n}\right)$ or $\operatorname{PSU}\left(3,2^{n}\right)$.

In 1973 building on work of Gorenstein and Walter and of Shult, Aschbacher A1] was able to strengthen Bender's Theorem to the precise 2-Uniqueness Theorem needed for the Signalizer Functor Method.

Paralleling these developments was another line of research which yielded dramatic surprises. Beginning early in the 1960's several researchers began to analyze simple groups with abelian Sylow 2-subgroups. It soon became clear that a crucial case was when the centralizer of an involution $z$ has the form:

$$
C_{G}(z)=\langle z\rangle \times L \cong \mathbf{Z}_{2} \times \operatorname{PSL}(2, q)
$$

for some $q \equiv \pm 3(\bmod 8)$. Thompson thought he could show that necessarily $q=3^{2 n+1}$, a case arising in the simple Ree groups ${ }^{2} G_{2}\left(3^{2 n+1}\right)$, but Janko Ja] discovered that one further case was possible: $q=5$. Janko's discovery of $J_{1}$ (a subgroup of $G_{2}(11)$ ), the first new sporadic simple group in a century, rivetted the attention of the group theory world and began a decade of feverish exploration and discovery in which 20 more sporadic groups came to light. The strategy of studying promising involution centralizers soon led Janko to two more simple groups with an isomorphic involution centralizer, and shortly thereafter rewarded Held, Lyons and O'Nan with simple groups. The construction of Janko's second group, $J_{2}$, by M. Hall as a rank 3 permutation group inspired a flurry of constructions of new simple groups by D. Higman and Sims, McLaughlin, Suzuki and Rudvalis (the latter a prediction, followed by a construction by Conway and Wales). Conway's investigations of the Leech lattice and Fischer's study of 3-transposition groups (discussed below) each led to three new sporadic simple groups.

A crowd of new Ph.D.'s entered the fray in the late 1960's and the classification project entered high gear. The level of excitement is captured in the language of Gorenstein's introduction to his book Finite Groups [G1], published in 1968:

"In the past ten years there has been a tremendous surge of activity in finite group theory. The period has witnessed the first serious classification theorems concerning simple groups and the discovery of several new families of simple groups; and, above all, the fundamental question of the solvability of groups of odd order has been answered. ... Out of the work of Feit and Thompson ... and Suzuki..., there is gradually emerging a body of techniques and a series of general methods for studying simple groups. Although the entire field is presently in an excited state of ferment and fluidity, as recent basic work of Glauberman and Alperin clearly indicates, a degree of stability appears to be settling over certain aspects of the subject."

In 1969, John Walter Wa achieved the reduction of the problem of groups with abelian Sylow 2-subgroups to the specific centralizer of involution problem which had been studied by Thompson, Janko and others. In this work he was forced to analyze the 2-signalizer problem in a context of nonsolvable involution centralizers. Soon he and Gorenstein began their deep analysis of the Signalizer 
Method, leading not only to the concept of a signalizer functor discussed above but to other fundamental concepts of balance and generation.

The search for a suitable replacement for the Fitting subgroup, which could be regarded as the "bottom" of a nonsolvable group led Gorenstein and Walter GW2 to the $p$-layer and extended $p$-layer. Inspired by comments of Gorenstein, Bender Be2 was led to the generalized Fitting subgroup.

Definitions. 1. A finite group $L$ is quasisimple if $L=[L, L]$ and $L / Z(L)$ is a simple group. A finite group $E$ is semisimple if $E$ is the commuting product of quasisimple groups.

2. If $H$ is a finite group, then $E(H)$ is the unique maximal normal semisimple subgroup of $H$. The generalized Fitting subgroup of $H$ is $F^{*}(H)=E(H) F(H)$.

3. If $H$ is a finite group, $p$ is a prime and $O_{p^{\prime}}(H)$ is the largest normal $p^{\prime}$ subgroup of $H$, then the $p$-layer of $H, L_{p^{\prime}}(H)$, is the subgroup of the full preimage of $E\left(H / O_{p^{\prime}}(H)\right)$ generated by $p$-elements.

The generalized Fitting subgroup $F^{*}(G)$ plays the role of the foundation on which $G$ stands, in the following sense:

Bender's $F^{*}$-Theorem. Let $H$ be any finite group. Then $F^{*}(H)$ is the commuting product of the semisimple group $E(H)$ and the nilpotent group $F(H)$. Moreover $F^{*}(H)$ contains $C_{H}\left(F^{*}(H)\right)$. Thus $H / Z(F(H))$ acts faithfully as a group of automorphisms of $F^{*}(H)$ and, in particular $|H| \leq\left|F^{*}(H)\right|$ !.

For the structure theory of finite groups, $F^{*}(G)$ is the key concept. On the other hand for the purpose of studying the embedding of a 2-local subgroup $H$ in a finite group $G$, the 2-layer of $H$ is more important, because it enjoys the following fundamental "balance" property, discovered by Gorenstein and Walter [GW3.

$L$-Balance Theorem (Gorenstein-Walter). Let $H$ be a 2-local subgroup of the finite group $G$. Then $L_{2^{\prime}}(H) \leq L_{2^{\prime}}(G)$.

This crucial result together with various extensions and modifications facilitates the comparison of the centralizers of two different commuting involutions in a finite group $G$. Its proof relies fundamentally on a weak version of Schreier's Conjecture, which was proved by Glauberman as a corollary of his $Z^{*}$-Theorem. A posteriori we know the truth of the full Schreier Conjecture and hence the validity of the analogous $L_{p^{\prime}}$-Balance Theorem for all primes $p$.

In addition to these major theorems, the Signalizer Functor Method requires a good choice of signalizer functor. A candidate functor was proposed by Gorenstein and Walter. Later Goldschmidt introduced a better functor, which was further modified and implemented by Aschbacher in his characterizations of simple groups of Lie type. By 1971 the stage was mostly set for the final attack on CFSG. Or was it?

\section{Gorenstein's Classification Program}

On the one hand major conceptual advances in the understanding of the local structure of finite simple groups had been achieved. On the other hand the great classification theorems of the 1960's from the Odd Order Theorem through the Alperin-Brauer-Gorenstein Theorem, while consuming almost 1,500 journal pages, had only completed the characterization of the groups $\operatorname{PSL}(2, q), P S L(3, q)$, $P S U(3, q), S z\left(2^{n}\right)$ and ${ }^{2} G_{2}\left(3^{n}\right)$ (the split $B N$-pairs of rank 1 in the language of 
Tits) and a few miscellaneous small groups: $A_{7}, M_{11}$ and $J_{1}$. (Indeed the Ree group characterization was not yet complete.) The vast ocean of finite simple groups remained still untamed and perhaps even still uncharted.

Already in 1971, Gorenstein proposed in a series of lectures in Israel a Classification Program. He refined this to a 16-step program (published in an appendix to [G3), which he announced during a summer group theory conference at the University of Chicago in 1972. In spite of the great strides of the previous decades, Gorenstein remained one of the few believers in the success of the Classification Project in our lifetimes. As he wrote later [G4]: "The program was met with considerable skepticism. I doubt that I made any converts at the time - the pessimists were still strongly in the ascendancy." This was to change dramatically in just a few years.

At the time of Gorenstein's lectures, the best-elaborated strategy dealt with groups of "odd type", i.e. groups of small 2-rank or groups in which $L_{2^{\prime}}\left(C_{G}(z)\right) \neq 1$ for some involution $z$. Indeed the "small odd type" case had just been completed by Gorenstein and Harada. (At the time groups in which $L_{2^{\prime}}\left(C_{G}(z)\right) \neq 1$ for some involution $z$ were called groups of component type. Later this became ambiguous as $p$-components in groups of characteristic 2-type were studied by Gorenstein and Lyons and the dichotomy odd type/even type emerged.)

The small case was Step I of Gorenstein's 16 steps. The signalizer analysis for the large odd-type case was Step II. It had been initiated by Gorenstein and Walter. The anticipated endpoint of the signalizer analysis was the following assertion, which was finally established by 1979 :

The $B$-Theorem. Let $G$ be a finite simple group. Then $L_{2^{\prime}}\left(C_{G}(z)\right)=E\left(C_{G}(z)\right)$ for every involution $z$ of $G$.

Steps IV, VI, VII and VIII dealt with various aspects of the Odd Type Case: the final identification problem for the groups of Lie type in odd characteristic, the alternating groups and most of the sporadic simple groups. A systematic treatment of this identification problem, given a precise involution-centralizer, had been initiated by Brauer, Fong, W. Wong and Phan. To get a feeling for such a problem in the easiest possible case (which actually leads not to a simple group, but to an almost simple group), it is instructive to consider the following problem (which is false for certain small $n$, as indicated below):

Problem. Suppose that $G$ is a finite group containing an involution $t$ such that $C_{G}(t)=\langle t\rangle \times L$, with $L \cong S_{n}$, the symmetric group on $n$ letters, $n \geq 5$. Suppose further that if $t_{i}$ is a transposition in $L$, then $C_{G}\left(t_{i}\right)=\left\langle t_{i}\right\rangle \times L_{i} \cong C_{G}(t)$. Prove that $G \cong S_{n+2}$.

When $n \geq 7$, it is easy to write down a set $\left\{t=t_{0}, t_{1}, t_{2}, \ldots, t_{n}\right\}$ of involutions with $t_{i}=(i-1, i) \in L$ for $2 \leq i \leq n$ and with $t_{1} \in C_{G}\left(t_{n}\right)$ and to verify all of the relations in the standard presentation for $S_{n+2}$ as a Coxeter group, except for the relation $t_{1} t_{n-1}=t_{n-1} t_{1}$. When $n$ is large enough, the final relation can be confirmed in the centralizer of some $t_{i}$, but when $n$ is small, the relation cannot be established. For example when $n=6$, the Weyl group of $E_{6}$ is an additional possibility. Yet another problem arises from the possibility that $G_{0}=\left\langle t_{0}, t_{1}, \ldots, t_{n}\right\rangle$ is a proper subgroup of $G$. This actually occurs when $n=10$ and $G=\operatorname{Aut}\left(F_{5}\right)$, the automorphism group of Harada's sporadic simple group, which contains $S_{12}$ as a proper subgroup. The latter problem can be analyzed using Aschbacher's 
extension of Bender's Strongly Embedded Theorem. The former problem bedevils the analysis of small cases. An analogous procedure applies to the groups of Lie type, using presentations by Steinberg or by Curtis and Tits in place of Coxeter presentations.

The starting point of the final identification step discussed above was detailed information about the structure of $C_{G}(z)$, or at least about $E\left(C_{G}(z)\right)$. The terminal point of the $B$-Theorem was important but vague information about $E\left(C_{G}(z)\right)$. There remained a substantial gap. The principal difficulty was the following. A priori $E\left(C_{G}(z)\right)$ could be the commuting product of arbitrarily many quasisimple "components". In most of the actual examples, the quasisimple components are stabilizers of eigenspaces for the semisimple linear operator $z$. Being an involution, $z$ can have no more than two eigenvalues, hence no more than two components. (In fact this assertion is false because of the fact that the derived subgroup of the orthogonal group $O^{+}(4, q)$ is a product of two quasisimple groups when $q>3$. Thus, most dramatically, $D_{4}(q), q$ odd, $q>3$, has an involution $t$ such that $E\left(C_{G}(t)\right)$ is the commuting product of four $S L(2, q)$ 's. But roughly speaking the assertion is true.) The problem was to provide an a priori proof of this observation, i.e. to bound the number of quasisimple components in the centralizer of some involution. This problem constitutes Steps III and V of Gorenstein's Program. Once that was achieved, in an inductive proof of the Classification Theorem, the possible isomorphism types of the components would be known from the list of conclusions of the Classification Theorem. Hence a finite number of families of Brauer-type involution-centralizer theorems would have to be proved.

A first version of such a bounding theorem was proved by Powell and Thwaites [PT]. Shortly thereafter, an optimal theorem was obtained by Aschbacher [A2, A3 in 1973.

Aschbacher's Component Theorem. Let $G$ be a finite simple group and suppose that the B-Theorem holds. Suppose that $E\left(C_{G}(t)\right) \neq 1$ for some involution $t$ of $G$. Then there exists an involution $z$ of $G$ and a quasisimple "standard" component $K$ of $C=C_{G}(z)$ such that $C_{C}(K)$ either has 2-rank 1 or is solvable with elementary abelian or dihedral Sylow 2-subgroups. In particular $C$ has at most two components and $K \triangleleft C$ unless $K$ has 2-rank 1 .

Now the logic of the Large Odd Type Case, as clarified by Aschbacher's work is:

Step 1. Prove the B-Theorem.

Step 2. Prove the Component Theorem.

Step 3. Solve the standard component problem for every possible quasisimple group $K$ such that $K / Z(K)$ arises as a conclusion of the Classification Theorem.

The chronology of the Large Odd Type Case on the other and was that Step 2 (by Aschbacher) preceded Step 1, while Step 3 was accomplished in a huge number of papers scattered from the early 1950's to 1981. Gorenstein's book [G5] is a detailed chronicle of this portion of the Classification proof. His bibliography contains 144 references, almost all crucial to this proof, and this omits many essential references from the bibliography of G4].

A strategy for the proof of the $B$-Theorem was developed by Aschbacher, Thompson and Walter at a conference in Sapporo in 1974 and was implemented by Aschbacher, Gilman, Harris, Solomon, Walter and others, primarily during the 1974-75 Group Theory Year at Rutgers University. (See [So] for a survey article.) A major ingredient in the proof is Aschbacher's characterization of the groups of Lie type 
over fields of odd order [A4], for which he was awarded the Cole Prize in Algebra. At this point the problem of groups of odd type was essentially "busted", although a substantial amount of mopping-up in terms of specific standard form problems was required and accomplished in the ensuing years by Seitz, Finkelstein, Gomi and others. (See [Se] for a survey article.)

Paralleling these developments was the exciting discovery of the Baby Monster and the Monster by Fischer and Griess in 1973, together with the intensive investigation of their subgroups by Thompson, Harada, Conway, Norton and others. In addition to four new simple groups, there emerged by 1979 the fascinating numerology of Monstrous Moonshine associated with the characters of the Monster and its subgroups. Early in 1974, Janko discovered $J_{4}$, once more by a judicious choice of involution-centralizer problem. With appropriate symmetry $J_{4}$ was to turn out to be the last of the sporadic simple groups to be unearthed.

Some additional words are in order about Fischer's work. Baer had returned to Germany in the mid 1950's, taking a post in Frankfurt, where he trained and inspired a generation (or two) of German algebraists, including Bender and Fischer. Fischer undertook the study of groups $G$ generated by a normal set $D$ of involutions whose pairwise products had restricted order. Baer (and independently Suzuki) had proved that if elements of $D$ pairwise generate 2-groups, then $\langle D\rangle$ is a 2group. Fischer considered the first new case, where the set of orders of $x y$ for $x, y \in D$ was $\{1,2,3\}$. He called the elements of $D$ 3-transpositions since the transpositions in $S_{n}$ have this property. In 1969 he succeeded in classifying simple 3 -transposition groups [Fi], discovering in the process three sporadic simple groups. Fischer's work became a major inspiration both for Aschbacher and for Fischer's own student, Timmesfeld. Aschbacher generalized Fischer's paper by classifying odd-transposition groups, in which any two non-commuting elements of $D$ have product of odd order. Timmesfeld [Tm1] studied $\{3,4\}^{+}$-transposition groups, where the set of orders is $\{1,2,3,4\}$ and if $x y$ has order 4 , then $(x y)^{2} \in D$, obtaining a characterization of most of the groups of Lie type in characteristic 2 in terms of root involutions. Fischer's pursuit of all $\{3,4\}$-transposition groups led him to the Baby Monster. The combinatorial and geometric nature of this study informed much of the thinking of Aschbacher and Timmesfeld, supplementing the arsenal of techniques deployed earlier by Thompson.

\section{Groups of CharaCteristic 2-Type}

The last eight steps of Gorenstein's program dealt with simple groups of characteristic 2-type, i.e. simple groups $G$ in which $F^{*}(H)$ is a 2-group for every 2-local subgroup $H$ of $G$. The prototypical examples are the groups of Lie type defined over finite fields of characteristic 2, but there are also a few sporadic examples, such as $\mathrm{Co}_{2}, J_{4}$ and $F_{3}$. With minor modification, Gorenstein's eight steps were in fact implemented: IX: Thin Groups (Aschbacher [A5]); X: Groups with a Strongly $p$-Embedded (2-Local) Subgroup, $p$ odd (Aschbacher [A8]); XI and XV: The Signalizer Functor Method and Component Theorem for Odd Primes (Gorenstein-Lyons [GL1]); XII: Groups of Characteristic $\{2, \mathrm{p}\}$-Type (Timmesfeld et al. Tm2]); XIII: Quasithin Groups (Mason Ms2]; Aschbacher-Smith [AS]); XIV Groups with $e(G)=3$ (Aschbacher [A7]); XVI Final Characterization of the Simple Groups of Characteristic 2-Type (Gilman-Griess GG]). I have taken some small liberties with the names of Gorenstein's steps. 
There was only one time that I saw Danny Gorenstein worried about the Classification Project. In the spring of 1975 , Janko wrote a letter to Danny announcing that he was giving up work on the Thin Group Problem, which he had been investigating for at least five years. Danny reported this to Thompson, who gave the problem some thought but abandoned it later that spring. Danny was worried. Maybe there was an impenetrable roadblock to the Classification.

What was the Thin Group Problem? In the N-Group Paper [T2] in which he classified simple groups all of whose local subgroups are solvable, Thompson introduced the parameter $e(G)$.

Definition. Let $G$ be a finite group. For $H$ a 2-local subgroup of $G$, denote by $e(H)$ the maximum rank of an abelian subgroup of $H$ of odd prime-power order. Then let $e(G)$ denote the maximum value of $e(H)$ as $H$ ranges over all 2-local subgroups of $G$.

By the Odd Order Theorem and a theorem of Frobenius, if $G$ is nonsolvable, then $e(G) \geq 1$. When $e(G)=1$, we call $G$ a thin group. Thus $G$ is thin if all odd-order Sylow subgroups of all 2-local subgroups of $G$ are cyclic.

The parameter $e(G)$ is of importance when $G$ is a simple group of characteristic 2 -type. The canonical examples for $G$ are the groups of Lie type in characteristic 2, and $e(G)$ roughly measures the twisted Lie rank (BN-rank) of $G$. Thus we would expect the thin simple groups of characteristic 2-type to be the groups $\operatorname{PSL}\left(2,2^{n}\right)$, $P S U\left(3,2^{n}\right)$ and $S z\left(2^{n}\right)$. These are indeed the "generic" examples, but a few additional examples occur. If $G$ has 2 -rank at least 3 , these are $P S L(3,4),{ }^{2} F_{4}(2)^{\prime}$ (the Tits group) and ${ }^{3} D_{4}(2)$. Aschbacher tackled this problem in the summer of 1975.

Starting in the early 1950's, even before Chevalley's constructions, Tits had studied geometries associated with Lie-type groups and by the mid-1960's had achieved an elegant and monumental theory of buildings, chamber systems and BN-pairs. Among other things, he classified [Ti] all finite irreducible BN-pairs of rank at least 3 (i.e. those in which the Weyl group is neither cyclic nor dihedral). In particular all finite simple BN-pairs of rank at least 3 are split, in the sense that $B=U(B \cap N)$ where $U$ is a nilpotent normal subgroup of $B$ and $U \cap N=1$. Fong and Seitz [FS] extended Tits' result by classifying split BN-pairs of rank 2. In particular every simple group $G$ of Lie-type defined over a finite field of characteristic $p$ is a split BN-pair with $U$ a Sylow $p$-subgroup of $G$ and with the BN-rank equal to the twisted Lie rank of $G$. Moreover the associated building is determined by the incidence relations among the so-called parabolic subgroups of $G$, i.e. the subgroups of $G$ containing a conjugate of $B$.

As the target groups for the Thin Group Theorem are all groups of Lie type in characteristic 2, it is natural to study the maximal subgroups of $G$ containing a Sylow 2-subgroup $U$. If there is a unique such maximal subgroup $M$, one would like to show that $G$ is a split BN-pair of rank 1 with $B=U(B \cap N)$, i.e. $G$ satisfies Suzuki's Theorem. In view of Bender's Theorem, it suffices to show that $N_{G}(D) \leq M$ for every nonidentity $D \leq U$. This is immediate by hypothesis if $D \triangleleft U$. In general one confronts a "pushing-up problem":

Problem. To show that if $S$ is a Sylow 2-subgroup of $N_{G}(D)$ with $S<U$, then there exists a nonidentity characteristic subgroup $C$ of $S$ with $C \triangleleft N_{G}(D)$.

If this is the case, then we can "push up" $N_{G}(D)$ to $N_{G}(C)$ which contains a larger 2-group than $S$, namely $N_{U}(C)$. Continuing in this manner one eventually 
pushes $N=N_{G}(D)$ up to a 2-local $N^{*}$ containing $U$. But then $N \leq N^{*} \leq M$ by hypothesis and Bender's Theorem may be invoked to identify $G$. By a theorem of Borel and Tits [GLS2, Theorem 3.1.1], this pushing-up process will always succeed if $G$ is a simple group of Lie type in characteristic 2 . However, there are almost simple examples where it fails, e.g. $G=L\langle\gamma\rangle$, where $L=P S L\left(3,2^{n}\right)$ and $\gamma$ is a graph automorphism of order 2 . This is at the heart of the difficulty of this problem.

Because of the thin hypothesis a Thompson factorization for $N=N_{G}(D)$ is essentially tantamount to the existence of the desired characteristic subgroup $C$. Hence in studying obstructions to pushing-up, one is led to confront failure-offactorization situations. A fundamental example occurs when $N / O_{2}(N) \cong$ $P S L\left(2,2^{n}\right)$ and $V / Z(N)$ is a natural module for $N / O_{2}(N)$, i.e. a $2 n$-dimensional irreducible $\mathbf{F}_{2}\left[N / O_{2}(N)\right]$-module. Baumann $[\mathrm{Ba}$ analyzed this situation and was able to determine the possible structures for $\mathrm{O}_{2}(N)$, introducing the Baumann subgroup which is crucial in circumventing the difficulty mentioned above. Glauberman and Niles [GN] extended his analysis. There still remained significant challenges, for example, the pushing-up problem when $N / O_{2}(N) \cong P S L(3,2)$. Moreover pushingup is only part of the problem. Given a pair of "parabolics" $\left(P_{1}, P_{2}\right)$ containing a given 2-Sylow normalizer $B$, Aschbacher must still show (given the hypotheses and prior results) that $G \cong{ }^{3} D_{4}(2)$. Nevertheless armed with these results and theorems of Thompson, Janko and Timmesfeld, Aschbacher succeeded in proving the Thin Group Theorem A5. He then continued to pursue the pushing-up problem, soon proving the Local $C(G, T)$-Theorem [A6] from which he, Foote and others deduced the Global $C(G, T)$ Theorem.

Global $C(G, T)$ Theorem. Let $G$ be a finite simple group of characteristic 2-type having 2-rank at least 3. Let $T$ be a Sylow 2-subgroup of $G$ and let $C(G, T)$ denote the subgroup of $G$ generated by the normalizers of all nonidentity characteristic subgroups of $T$. If $C(G, T)<G$, then $C(G, T)$ is a strongly embedded subgroup of $G$ and so $G \cong S L\left(2,2^{n}\right), S z\left(2^{n}\right)$ or $P S U\left(3,2^{n}\right)$.

Aschbacher's accomplishments in the years 1974-75 were staggering in their depth and breadth. Belief mounted that nothing could stand between him and the completion of the Classification.

Meanwhile Gorenstein and Lyons had begun their attack on the generic simple groups of characteristic 2-type. Their goal was to characterize the simple groups of Lie-type over fields of order $2^{n}$ and their strategy was modelled on the strategy developed by Gorenstein and Walter for the odd-type case. Their philosophy was that the significance of the prime 2 in the odd-type context was not its evenness but the fact that involutions are semisimple elements in groups of Lie type over fields of odd order; indeed usually they even lie in a split torus. This dictates the structure of centralizers of involutions which in turn dictates the shape of the proof. Thus when the target group is a group of Lie type in characteristic 2, one should still study the centralizers of semisimple elements of $G$; only these elements will now have odd order. This strategy in essence goes back to Killing and the search for a maximal toral subalgebra in a semisimple Lie algebra. It entails the choice of changing primes from 2 to a suitable odd prime which would divide the order of a split torus in $G$ if possible. (Of course $G$ is not known a priori to come from an algebraic group. So "split torus" is meaningless. However, with the Lie type examples in mind, Gorenstein and Lyons choose $p$ to maximize the 2-local $p$-rank of $G$, modifying this choice later in the analysis if necessary.) 
Many parts of the analysis do indeed parallel the corresponding parts of the odd-type analysis. There are liabilities however. Two involutions in $G$ generate a dihedral subgroup of $G$. Two elements of order $p$ in $G$ probably generate $G$. This undermines the counting arguments which had been exploited to great effect by Brauer, Feit, Suzuki and Bender. In particular it seems impossible to give an a priori proof of the odd analogue of Bender's Strongly Embedded Theorem. (A posteriori there is a Strongly $p$-Embedded Subgroup Theorem for all primes $p$ not dissimilar to Bender's theorem for $p=2$. In particular it asserts that if $G$ is a finite simple group of $p$-rank at least 3 having a strongly $p$-embedded subgroup, then $G$ is a split $B N$-pair of $B N$-rank 1 in characteristic $p$.) Inasmuch as one fork of the Gorenstein-Walter Alternative which underlies Signalizer Functor Analysis is the existence of a strongly $p$-embedded subgroup, this is a serious liability. However, in the context of their analysis of centralizers of semisimple elements in simple groups of characteristic 2-type, Gorenstein and Lyons [GL1] were able to refine the Gorenstein-Walter dichotomy to the following Trichotomy Theorem, which completed Steps XI and XV of Gorenstein's Program and formed the structural capstone of the characteristic 2-type portion of the Classification:

Gorenstein-Lyons Trichotomy Theorem. Let $G$ be a simple group of characteristic 2-type with $e(G) \geq 4$ in which all proper subgroups have known simple composition factors. Then one of the following alternatives holds:

1. There is an odd prime $p$ and an element $x$ of $G$ of order $p$ such that $C_{G}(x)$ has a normal quasisimple subgroup $L$ with $L / Z(L)$ a group of Lie type in characteristic 2; or

2. G has a maximal 2-local subgroup $M$ which is a p-uniqueness subgroup for some odd prime $p$ such that $M$ has p-rank at least 4 ; or

3. $G$ is of $G F(2)$-type.

Some clarification and explanation of the terminology in this theorem is in order. First, conclusion (1) in fact contains quite a bit more information than is stated above concerning centralizers of certain elements $y$ of order $p$ commuting with $x$. Indeed there is enough information to construct a large subgroup $G_{0}$ of $G$ via the Steinberg or Curtis-Tits relations for groups of Lie type and then to show that $G=G_{0}$ using refinements of Bender's Strongly Embedded Theorem. This was accomplished by Gilman and Griess [GG], completing Step XVI.

In case (2), often called the Uniqueness Case, $M$ is roughly a strongly $p$-embedded subgroup of $G$. The key extra fact is that $M$ is a 2-local subgroup of $G$. Indeed it may be shown that $M$ contains a Sylow 2-subgroup of $G$. By the Global $C(G, T)$ Theorem there must be another 2-local subgroup $N$ of $G$ such that $M \cap N$ contains a Sylow 2-subgroup of $G$. This is the point of departure for the analysis of case (2). The problem is still extremely difficult. Nevertheless it was solved by Aschbacher A8], completing Step X.

It remains to explain $G F(2)$-type. Although the only simple groups which satisfy the hypotheses of the Trichotomy Theorem are finite groups of Lie type in characteristic 2, there are certain near misses associated primarily with the sporadic simple groups for which neither conclusion (1) nor conclusion (2) holds. Rather, a variant of (1) holds in which $L / Z(L)$ is a group of Lie type in characteristic $p$ or a sporadic simple group. This should be regarded as the natural extension of a case first considered by Thompson in the N-Group Paper and later treated more generally by Klinger and Mason [KM], namely the case where $G$ is both of characteristic 
2-type and of characteristic $p$-type for $p$ an odd prime such that some 2-local has $p$-rank at least 2. As such, this is Step XII of Gorenstein's Program. An easy first step of the analysis leads to the conclusion that for some 2-local subgroup $H$ of $G$, $F^{*}(H)$ is a 2-group of symplectic type in the sense of Philip Hall.

Definitions. 1. A 2-group $T$ is of symplectic type if every characteristic abelian subgroup of $T$ is cyclic. It follows that $T=E \circ R$, where $E$ is an extraspecial 2 -group (or $E=1$ ) and $R$ is cyclic or of maximal class.

2. A group $G$ is of $G F(2)$-type if for some involution $t$ of $G, F^{*}\left(C_{G}(t)\right)$ is a 2 -group of symplectic type.

Sixteen of the sporadic simple groups are of $G F(2)$-type and indeed this feels like the source of all sporadic groups. Thus it was a shock when Timmesfeld [Tm2 announced in 1976 the local classification of all simple groups of $G F(2)$ type, the global determination following soon thereafter by work of S. D. Smith and others. We could hear the bell toll for the Classification. At the Duluth Group Theory Conference in the summer of 1976 the energy which had driven the classification endeavor turned into a frenzy akin to the Oklahoma land rush, as researchers scrambled to lay claim to the few remaining problems. Foremost among the unresolved problems were the cases $e(G)=2$ and 3 (Steps XIII and XIV), which lay between Aschbacher's Thin Group Theorem and the Gorenstein-Lyons Trichotomy. The former was claimed by G. Mason and the latter by Aschbacher. The $e(G)=2$ case had been dubbed the Quasithin Case by Janko.

Psychologically the Classification was over in 1976. As recently as 1974 in the wake of the discoveries of $J_{4}$, the Monster and its subgroups, Alperin had remarked that the Classification seemed to be converging and diverging at equal rates. But in a talk on October 30, 1976, Brauer reported [Br2]:

"It seems that most group theorists feel that it is only a matter of time until all finite simple groups will be classified. Jonathan Alperin wrote to me recently: 'It is a good guess that within five years everything should be pretty clear. But how long it will take to clean up and correct all the papers - and they do need that - is anybody's guess.'”

When long manuscripts were circulated a few years later by Aschbacher [A7, Mason [Ms2], and Gilman and Griess [GG] treating all the outstanding problems, it was natural for Gorenstein to declare the Classification Project completed in 1981, even though many difficult and fundamental papers had yet to wend their way through the refereeing and publication process. By the end of 1983 all of the relevant papers had indeed been published, except for Mason's quasithin manuscript, which was to remain an almost-completed, never-published torso.

\section{The LONG GOODBYE}

The proceedings of the 1978 Research Symposium in Finite Simple Groups [Cl] held in Durham, England, were published in 1980. The preface by Collins expresses the basic perception reinforced by the text: "The dominant area of discussion was the classification of simple groups, a programme which is now almost complete." Indeed there are essays by many of the major researchers summarizing the status of the important subproblems. The classification of groups of component-type (odd-type) is reported by Seitz and Solomon to be complete modulo two specific characterization problems, solved soon thereafter by Aschbacher and Walter. All other major theorems are covered in articles by Aschbacher, Gorenstein, Griess, 
Mason and Timmesfeld, with the exception of the $e(G)=3$ problem, whose solution by Aschbacher is reported by Collins in his introductory survey article. When the finite group theorists gathered in Santa Cruz, California, the following summer (1979) for a long conference, it was perceived as a wrapping-up of the classification project and a charting of new directions for the field. A (false) rumor circulated before the conference that Philip Hall, a notorious recluse, planned to attend, leading Graham Higman to quip that it was likely since Philip Hall liked to attend funerals.

There remained a gap concerning the existence and uniqueness of some of the sporadic simple groups, notably the Monster. However, in January 1980 Griess startled everyone by announcing a computer-free construction of the Monster $\mathrm{Gr}$. Over the previous decade Sims had developed powerful methods for the computer construction of finite simple groups as permutation groups, achieving striking successes beginning with the construction of the Lyons group as a permutation group of degree almost 9 million and culminating in the construction (jointly with Leon) of the Baby Monster as a permutation group of degree approximately 14 billion. But the Monster was daunting even for Sims and his computer. Griess avoided this problem by instead defining a non-associative commutative algebra of dimension 196884 having the Monster as its group of automorphisms. Investigations associated with the Griess algebra and its associated vertex operator algebra have stimulated some of the most fruitful research of the post-Classification era, including the Fields Medal research of Borcherds. Uniqueness questions for some of the sporadic groups such as the Monster remained open for quite a few years. Nevertheless it appeared in 1980 that one could claim the following theorem:

The Classification Theorem. Let $G$ be a finite simple group. Then $G$ is either

(a) a cyclic group of prime order;

(b) an alternating group of degree $n \geq 5$;

(c) a finite simple group of Lie type; or

(d) one of 26 sporadic finite simple groups: the five Mathieu groups, the four Janko groups, the three Conway groups, the three Fischer groups, HS, Mc, Suz, Ru, He, Ly, ON, HN, Th, BM and $M$.

(As uniqueness theorems trickled in, statement (d) became precisely accurate. For example, Griess, Meierfrankenfeld and Segev established the uniqueness of the Monster in [GMS].) In an unpublished draft of the preface to his Finite Simple Groups: An Introduction to Their Classification [G4], Gorenstein asserts: "In August, 1980, the classification of the finite simple groups was completed." In the published version, he amended this to February 1981. Gorenstein attributed the final theorem to Norton, while Aschbacher attributed the final theorem to me. Both were inaccurate. A gap remained to be filled. Around 1989 Aschbacher noticed that Mason's 800-page manuscript on quasithin groups was incomplete in various ways; in particular it lacked a treatment of certain "small" cases. By 1992 Aschbacher had prepared a manuscript treating the remaining cases and announced his result at the Joint AMS/LMS meeting that year. However, both manuscripts remain unpublished. Finally in 1996 Aschbacher and Smith took on the task of proving and publishing a proof of the Quasithin Theorem. This has been no small task. It is important to understand that in finite group theory, as in some other areas of mathematics, bigger is in many ways easier. Signalizer functors are available when the $p$-rank is at least 3 , not when it is 1 or 2 . Curtis-Tits presentations exist when the $B N$-rank is at least 3 , not when it is 1 or 2 . In an inductive setting, groups 
whose proper subgroups have composition factors which are large (known) simple groups are more manageable than groups whose proper subgroups have only abelian or very small simple composition factors. Most large simple groups have a unique "identity". Many small simple groups have multiple personalities, for example:

$$
\begin{aligned}
& A_{5} \cong S L(2,4) \cong P S L(2,5) \cong O(3,5)^{\prime} \cong O^{-}(4,2)^{\prime} ; \\
& A_{6} \cong P S L(2,9) \cong M_{10}^{\prime} \cong O(3,9)^{\prime} \cong O^{-}(4,3) \cong S p(4,2)^{\prime} ; \\
& P S p(4,3) \cong O(5,3)^{\prime} \cong P S U(4,2) \cong O^{-}(6,2)^{\prime} \cong W\left(E_{6}\right)^{\prime} .
\end{aligned}
$$

Moreover the structures of outer automorphism groups and Schur multipliers are uniformly described for all large simple groups, while numerous anomalies arise for small simple groups. These facts and others are instrumental in the existence of the sporadic simple groups, many of which are quasithin, and plague the analysis of quasithin groups.

Nevertheless the Aschbacher-Smith manuscript is near completion (see http:// www.math.uic.edu/smiths/papers/quasithin/quasithin.dvi) and with its publication by the A.M.S. will come the true completion of the classification of the finite simple groups.

\section{REVISIONS}

New approaches to old problems began early. Indeed Glauberman's $Z J$-Theorem may be regarded as a revision of Thompson's factorization theorems and was certainly used as such in Bender's revised proof of the Feit-Thompson Uniqueness Theorem. Also Dade Da] improved the coherence map for the character theory part of the Odd Order Paper.

The Uniqueness Theorem was the beginning of Bender's role as the quintessential revisionist. Shortly thereafter $\mathrm{Be} 2$ he dramatically shortened the proof of Walter's theorem on groups with abelian Sylow 2-subgroups, introducing the generalized Fitting subgroup and proving the following theorem.

Bender's Maximal Subgroup Theorem. Let $G$ be a finite simple group with distinct maximal subgroups $M$ and $N$ such that $F^{*}(M) \leq N$ and $F^{*}(N) \leq M$. Then there exists a prime $p$ such that both $F^{*}(M)$ and $F^{*}(N)$ are p-groups.

Indeed if $G$ is a finite simple group of Lie type in characteristic $p$ and $M$ and $N$ are two maximal parabolic subgroups of $G$ containing the same Sylow $p$-subgroup of $G$, then $M$ and $N$ satisfy the hypotheses (and conclusion) of Bender's theorem. The theorem is particularly effective in passing from the case where $F^{*}(M)$ is nilpotent to the characteristic $p$-type case. Bender's methods were soon adopted by Goldschmidt to help prove the 2-Signalizer Functor Theorem and to classify groups with a strongly closed abelian 2-subgroup [Go4]. Bender himself applied his methods to the revision of the Gorenstein-Walter theorem on groups with dihedral Sylow 2-subgroups. This work was largely completed by 1972 but remained unpublished for a decade, in part because of Bender's reluctance to use modular character theory. Finally Glauberman showed Bender how to replace the modular character theory with ordinary character theory and the work was published [BG1], [Be4].

Meanwhile in the late 1970's Glauberman and Bender had been thinking independently about further revision of the local analysis of the Odd Order Paper, and Glauberman brought a completed manuscript to Santa Cruz in 1979. Bender,

however, had additional improvements and again final publication was delayed for 
a decade. Around the same time Sibley was finding further improvements of Feit's coherence results and prepared extensive notes for a revised treatment of the character theory portion of the Odd Order Theorem. Finally Peterfalvi found a slick proof of the final generator and relations argument of the Odd Order Paper and, using the earlier work of Dade and Sibley, obtained a revised proof $\mathrm{Pe} 2$ of complementary material to the Bender-Glauberman Local Analysis for the Odd Order Theorem [BG2]. Peterfalvi also obtained a revised proof of Suzuki's Theorem on 2transitive groups, as well as O'Nan's characterization of $P S U(3, q)$ for $q$ odd [Pe1]. With Enguehard's treatment [E] of the work of Thompson, Bombieri and others on the Ree groups, this constituted a complete treatment of the foundational work on groups of odd order and split BN-pairs of rank 1.

Struck by the elegance of the Bender-Glauberman work on groups with dihedral Sylow 2-subgroups, I began in 1981 to investigate the possibility of extending their methods to treat groups with semidihedral Sylow 2-subgroups. At about the same time, Gorenstein and Lyons initiated a major project to prepare a complete unified proof of the Classification Theorem modulo the foundational theorems mentioned in the previous paragraph and modulo the existence, uniqueness and basic properties of the sporadic simple groups. In 1982 I joined their team (GLS) and extended the scope of my particular focus to cover the so-called Small Odd Case. Meanwhile Gorenstein and Lyons focussed their primary attention on extending the work in their Memoirs volume (on groups of characteristic 2-type with $e(G) \geq 4$ ) to cover so-called Generic Groups, both of odd-type and of even-type.

A few words are in order concerning the odd-type/even-type dichotomy. One of the fundamental consequences of Thompson's work was to shift attention from the top of a group (the domain of the transfer map) to the bottom. It is useful to think of the major intermediate results of the Classification not as theorems about simple groups but rather as local criteria for the triviality or simplicity of certain characteristic subgroups of an arbitrary finite group $G$, just like Cartan's Criterion for the triviality of the solvable radical of a Lie algebra. Thompson's ideas provided powerful new tools for proving such criteria for characteristic subgroups of $F(G)$ (later $F^{*}(G)$ ). Thus the $B$-Theorem and its analogues establish from local data the fact that $F(G)=1$, so that $F^{*}(G)=E(G)$ is a direct product of nonabelian simple groups. Next Aschbacher's Component Theorem and its analogues establish from local data that in fact $F^{*}(G)$ is a single nonabelian simple group. However, none of these theorems enables one to detect that $G=F^{*}(G)$, i.e. to detect locally that $\operatorname{Aut}(E)$ is not a simple group, where $E$ is a nonabelian simple group. Of course this is precisely where transfer theorems come into play (in view of the Schreier Conjecture), but it is perhaps better to think of the Classification Theorem as a classification of finite groups $G$ such that $F^{*}(G)$ is a nonabelian simple group. From this point-of-view, the groups of characteristic 2-type should be those finite groups $G$ such that $F^{*}(G)$ is a nonabelian simple group of Lie type in characteristic 2 . But now the internal criterion that $F^{*}(H)=\mathrm{O}_{2}(H)$ for every 2-local subgroup $H$ of $G$ is no longer the appropriate one. For if $t$ is an involution in $G$ inducing a graph, field or graph-field automorphism on $F^{*}(G)$ and $H=C_{G}(t)$, then usually $E(H) \neq 1$. Instead $E(H)$ is again a simple group of Lie type in characteristic 2 . The notion of even-type is a weakening of the notion of characteristic 2-type in such a way that almost all finite groups $G$ in which $F^{*}(G)$ is a simple group of Lie type in characteristic 2 are groups of even type. An interesting and not unattractive 
consequence of this is that most of the sporadic simple groups are groups of eventype, although only a few are groups of characteristic 2-type. There is a broad consensus on the desirability of replacing characteristic 2-type with some version of even-type as described here. There is much less agreement on the ideal definition of even-type. One version is given in [GLS1]. A different version (called "even characteristic" to avoid confusion) is given by Aschbacher and Smith. Yet another version has been proposed by Meierfrankenfeld.

Groups of characteristic 2-type have their analogues in groups of characteristic $p$-type for all primes $p$. And just as for 2 , this notion can be weakened to a notion of $p$-type which captures almost all finite groups $G$ for which $F^{*}(G)$ is a simple group of Lie type in characteristic $p$. Again many sporadic simple groups are of $p$-type for at least one odd prime $p$. Indeed the property of $G$ being both of 2-type (even type) and $p$-type for some odd prime $p$ such that the 2-local $p$-rank of $G$ is at least 2 is practically a defining property of the sporadic simple groups. The first theorem in this vein was proved by Thompson as part of his $N$-Group Paper. This was generalized in a paper of Klinger and Mason. An unpublished manuscript of Gorenstein and Lyons GL2] characterizes five of the sporadic simple groups $\left(\mathrm{Co}_{1}\right.$, $F i_{23}, F i_{24}^{\prime}, B M$ and $M$ ) in this spirit.

In the late 1970's Goldschmidt began to rethink the pushing-up theorems of Baumann, Glauberman and Niles. He was inspired by earlier work of Sims, who had used a graph-theoretic result of Tutte to prove a pushing-up result for groups $M$ with $M / O_{2}(M) \cong S_{3}$. Goldschmidt's analysis evolved into a strategy for studying groups $G$ generated by a pair of subgroups $\left(L_{1}, L_{2}\right)$ such that

1. $L_{1} \cap L_{2}$ contains a common Sylow 2-subgroup of both $L_{1}$ and $L_{2}$, and

2. $G$ has no nontrivial normal 2-subgroup.

Instead of studying a finite group $G$ with these properties, Goldschmidt considered the free product of $L_{1}$ and $L_{2}$ amalgamated over the subgroup $L_{1} \cap L_{2}$ acting on its associated tree. This provided a supple new language for Thompson's weak closure analysis. Stroth and Stellmacher soon followed in Goldschmidt's footsteps, and his strategy was refined and dubbed the Amalgam Method. Goldschmidt's initial motivation was to provide a new approach to results like Thompson's $N$ Group Theorem and Aschbacher's Thin Group Theorem, and indeed these two goals were accomplished by Stellmacher [S12],[S13]. A central accomplishment was the classification of weak $B N$-pairs of rank two by Delgado and Stellmacher [DGS, substantially extending Goldschmidt's original work on automorphism groups of trivalent graphs G05. One typical feature of these theorems is that it leads to the identification up to isomorphism of the amalgam $\left(L_{1}, L_{2} ; L_{1} \cap L_{2}\right)$, not the possible faithful finite completions of the amalgam. Indeed the classification of all faithful finite completions is often infeasible. However, if the finite group $G$ is assumed to be of characteristic 2-type and if $L_{1} \cap L_{2}$ is assumed to contain a Sylow 2-subgroup $S$ of $G$, then it is easy to see (by the Brauer-Fowler Theorem) that $|G|$ is bounded by $|S|$ and so the problem becomes finite and indeed manageable. In fact a program of characterizations of this type was initiated by Suzuki in the 1960's. Indeed an attack on the Quasithin Theorem by somewhat similar methods was begun in the 1970's by Gomi, later joined by Hayashi and others. Stellmacher also studied the Quasithin Problem via the Amalgam Method, but in the course of the 1990's his focus shifted to the general theory of groups of characteristic $p$-type. Joined first by his student, Meierfrankenfeld, and later by Stroth, Chermak, Parmeggiani, Parker 
and Rowley, yet another major revision project $\left(M C P^{2} R S^{2}\right)$ took shape, more or less orthogonal to the GLS project.

The goal of the GLS project is to classify finite simple groups $G$ for which there exists a prime $p$ such that $G$ has $p$-rank at least 3 and $G$ is NOT of $p$-type, choosing $p=2$ if possible. In addition we also plan to classify simple groups of 2-rank 2 , as well as simple groups which are both of even type and of $p$-type for some odd prime $p$ such that $G$ has 2-local $p$-rank at least 3. When these conditions fail, either $G$ has odd order or $G$ is of quasithin even type. In either case we (will) have other theorems to invoke (Feit-Thompson and Aschbacher-Smith).

On the other hand the goal of the $M C P^{2} R S^{2}$ project is to classify finite simple groups $G$ which are of characteristic $p$-type for some prime $p$ and which are generated by the $p$-local overgroups of some fixed Sylow $p$-subgroup of $G$. For a suitably weakened interpretation of the term characteristic $p$-type, this should cover all finite simple groups except for the alternating groups, $J_{1}$, and the split $B N$-pairs of rank 1. The $M C P^{2} R S^{2}$ team does not at present have an independent strategy for identifying these groups. Nevertheless their approach might yield a more efficient treatment of groups of even type than that of the GLS and Aschbacher-Smith teams.

A rough count by Gorenstein showed that the original proof of the Classification Theorem occupies about 15,000 journal pages. By incorporating new ideas, efficiencies of organization and the systematic use of the hypothesis that $G$ is a minimal counterexample to the Classification, the GLS proof with its numerous supporting results should occupy about 5,000 printed pages, a substantial reduction but still a daunting length. Although new ideas within the same conceptual framework might well prune hundreds of pages from the proof, it is difficult to imagine an orderof-magnitude reduction in the length of the proof without abandoning the entire approach and perhaps moving beyond the category of finite groups.

Is there a completely new and revolutionary approach to the Classification waiting to be discovered? In Thompson's eloquent article "Finite Non-Solvable Groups" written around 1982 [T4], he says:

"... the classification of finite simple groups is an exercise in taxonomy. This is obvious to the expert and to the uninitiated alike. To be sure, the exercise is of colossal length, but length is a concomitant of taxonomy. Those of us who have been engaged in this work are the intellectual confreres of Linnaeus. Not surprisingly, I wonder if a future Darwin will conceptualize and unify our hard won theorems. The great sticking point, though there are several, concerns the sporadic groups. I find it aesthetically repugnant to accept that these groups are mere anomalies...Possibly... The Origin of Groups remains to be written, along lines foreign to those of Linnean outlook."

I doubt that any developments of the past two decades would change Thompson's summary of the state of the field. We are still waiting and wondering. Are the finite simple groups, like the prime numbers, jewels strung on an as-yet invisible thread? And will this thread lead us out of the current labyrinthine proof to a radically new proof of the Classification Theorem?

\section{Applications AND other DeVelopments}

What next? It is easier to recount the past than to predict the future. In the immediate aftermath of the announcement of the Classification there was intense 
activity culling the more immediate consequences of the theorem by verifying old conjectures in the theory of groups, geometries and elsewhere. Venerable conjectures such as Schreier's Conjecture and Frobenius' Conjecture were confirmed fairly quickly. Likewise the classification of $k$-transitive permutation groups $(k \geq 2)$ was completed along with several other classifications of classes of permutation groups or geometric groups. These results in turn made possible the determination of the Galois groups of numerous classes of polynomial equations. In contrast to these taxonomic theorems, Borovik, Cherlin and others have used some of the fundamental methods of the Classification proof in their investigation of infinite simple groups of finite Morley rank.

Perhaps the most dramatic corollary of the Classification is the affirmative solution of the Restricted Burnside Problem. The Hall-Higman paper [HH] had reduced the Restricted Burnside Problem to the prime-power exponent case, which was settled by Zelmanov, together with certain assertions about finite simple groups which are easy corollaries of the Classification. The theorem has also influenced thinking about the Inverse Galois Problem, though this remains open and indeed seems far from being reduced to the case of finite simple groups.

The field of ordinary and modular representations of finite groups (not to mention integral representations) remains full of difficult problems and intriguing conjectures (by Brauer, Donovan, Alperin, Dade, Broué, Puig and others) in spite of both the Classification and the major achievements of Lusztig and others on the representation theory of finite groups of Lie type. A plethora of new ideas and approaches has emerged involving the theory of algebras, derived categories, subgroup complexes, algebraic geometry and algebraic groups, infinite-dimensional representations and more.

The classification of primitive permutation representations (equivalently, maximal subgroups) of the finite simple groups was launched as a program at the Santa Cruz Conference by L. Scott, who co-contributed the O'Nan-Scott Theorem on maximal subgroups of $S_{n}$, later supplemented by Aschbacher's Theorem on maximal subgroups of classical linear groups. Some of the most difficult questions have stimulated deep investigations of the interaction between finite and algebraic groups by Seitz, Testerman, Liebeck and others, yielding major dividends for algebraic group theory and representation theory as well and continuing to generate new questions, for example, concerning tilting modules.

The flag-transitive action of groups on geometries, extending Tits' theory of buildings, was initiated by Buekenhout and pursued energetically by many researchers, including Aschbacher, Timmesfeld, Ronan, Smith, Stroth, Ivanov and Shpectorov. This has led to new characterization theorems for many of the sporadic groups, as well as the groups of Lie type, motivating the proposed endgame for the $\mathrm{MCP}^{2} \mathrm{RS}^{2}$ program. In related work, Tits and Weiss have classified Moufang generalized $n$-gons, and numerous applications to graph theory have been obtained by Praeger, Saxl and many others. A still elusive will-o'-the wisp is an elegant set of axioms, in the spirit of Tits' axioms for a building, defining a class of geometries for all of the finite simple groups and perhaps more, but not too much more.

Monsterology has become its own subfield, spawning the mathematical investigation of vertex operator algebras by Lepowsky, Borcherds, Miyamoto, Griess, Mason and others. Again questions abound, ranging from a foundational search for the "correct" definition of a VOA to the search for a truly satisfying explanation of Monstrous Moonshine. In addition to Moonshine, numerous Monster-related 
mysteries have been observed by McKay, Glauberman, Norton, Lyons and others, including many strange parallels with the exceptional groups of Lie type. Pushing through the envelope of finite groups, Conway has constructed a groupoid $M_{13}$ containing both $M_{12}$ and $P S L(3,3)$, while Dwyer and Wilkerson have constructed an $\mathrm{H}$-space, $\mathrm{DI}(4)$ related to $\operatorname{Spin}(7)$ and the Conway group $\mathrm{Co}_{3}$. The octonions and the exceptional simple Jordan algebra have well-established connections to the exceptional Lie groups, but also to the Leech lattice. I believe there is much gold still to be mined in this vein.

An ever-improving arsenal of computer algorithms for the identification of permutation groups, linear groups and more generally "black box" groups is being assembled by Kantor, Seress and many other researchers. In particular there is hope of determining all finite groups of order at most 2001 in the year 2001. On the other hand, calculation of groups of order $2^{10}$ has reconfirmed the long-known fact that "most" finite groups are nilpotent groups of nilpotence class at most 2 and they exist in frightening numbers.

Thus the classification of all finite groups is completely infeasible. Nevertheless experience shows that most of the finite groups which occur "in nature" - in the broad sense not simply of chemistry and physics, but of number theory, topology, combinatorics, etc.- - are "close" either to simple groups or to groups such as dihedral groups, Heisenberg groups, etc., which arise naturally in the study of simple groups. And so both the methodologies and the database of information generated in the course of the Classification Project remain of vital importance to the resolution of questions arising in other disciplines.

Is the database correct? Is there a 27th sporadic simple group? I seriously doubt it, but it would be chutzpahdich to assert that a 5000-page 40-year human endeavor is beyond the possibility of human error. It is heartening that the Revisionists have scrutinized and rethought the delicate and difficult work on groups of odd order and split $B N$-pairs of rank 1. The Aschbacher-Smith and $M C P^{2} R S^{2}$ projects give promise of providing two independent proofs of the Quasithin Theorem. The GLS team is carefully reworking the rest, and we are most grateful that Thompson, Ho and Sin have been studying our manuscripts. For almost two decades in the 60's and 70's, keen-eyed seekers scoured the terrain searching for new sporadic treasures. All of this bolsters our confidence. But finally we await the visionaries of new generations, the Darwins of Thompson's metaphor, who will shed unexpected new light on this ever-fascinating subject. Hilbert dreamed of returning in 100 years to ask about the Riemann Hypothesis. I would like to return in 100 years and ask: "What do the sporadic simple groups really mean?" As Hilbert would say: We must know! We will know!

\section{Personal Remarks and acknowledgements}

In the summer of 1970 I participated in an exhilarating NSF-funded Summer Institute on Finite Groups at Bowdoin College. During that summer and the years immediately following it, I had the rare privilege to work with and befriend some of the best mathematicians and finest human beings anyone could ever hope to meet, including but not limited to: Walter Feit, David Goldschmidt, Richard Lyons, Leonard Scott, Danny Gorenstein, Koichiro Harada, Jon Alperin, George Glauberman and Paul Fong. I owe them all, and of course John Thompson, Helmut Bender and Michael Aschbacher, a debt which I could never begin to repay. It has been 
the gift of these teachers that I have been able to appreciate the beauty of their ideas. Even more it has been a privilege to know them as friends and share my life with them. The group theory community has been a blessed place to work, and I can only acknowledge with deepest gratitude the nobility of spirit of all those great mathematicians who have made it that way.

With specific regard to this article, my understanding of the history of group theory has benefitted greatly from my acquaintance with Peter Neumann, and the discussion of the period 1890-1910 in this article relies heavily on a survey article of his to appear in the forthcoming Collected Works of William Burnside. Thompson's article [T4 is a fascinating document and is quoted extensively above. My view of the history of the Classification is of course indelibly colored by Gorenstein's numerous surveys and personal recollections such as G6. My understanding of Suzuki's place in the saga has grown considerably thanks to conversations with Harada and a fascinating article of his to appear shortly [Hr2]. I thank Steve Smith and Inna Korchagina for their careful scrutiny of this article, as well as Aschbacher and Harada for their comments. G.E. Wall, Huppert and Alperin also deserve thanks for their private communications. And always a special wink and a smile for Richard Lyons.

\section{REFERENCES}

[Al] J. L. Alperin, Sylow intersections and fusion, J. Algebra 6 (1967), 222-241. MR 35:6748

[ABG1] J. L. Alperin, R. Brauer and D. Gorenstein, Finite groups with quasi-dihedral and wreathed Sylow 2-subgroups, Trans. Amer. Math. Soc. 151 (1970), 1-261. MR 44:1724

[ABG2] J. L. Alperin, R. Brauer and D. Gorenstein, Finite simple groups of 2-rank two, Scripta Math. 29 (1973), 191-214. MR 53:5728

[A1] M. Aschbacher, Finite groups with a proper 2-generated core, Trans. Amer. Math. Soc 197 (1974), 87-112. MR 51:681

[A2] M. Aschbacher, On finite groups of component type, Illinois J. Math. 19 (1975), 78-115. MR 51:13018

[A3] M. Aschbacher, Tightly embedded subgroups of finite groups, J. Algebra 42 (1976), 85-101. MR 54:10390

[A4] M. Aschbacher, A characterization of Chevalley groups over fields of odd order. I, II, Ann. of Math. 106 (1977), 353-468. MR 58:16865a; MR 58:16865b

[A5] M. Aschbacher, Thin finite simple groups, J. Algebra 54 (1978), 50-152. MR 82j:20032

[A6] M. Aschbacher, A factorization theorem for 2-constrained groups, Proc. London Math. Soc. (3) 43 (1981), 450-477. MR 83a:20019b

[A7] M. Aschbacher, Finite groups of rank 3, I, Invent. Math. 63 (1981), 357-402; II, Invent. Math. 71 (1983), 51-163. MR 82j:20033; MR 84h:20008

[A8] M. Aschbacher, The uniqueness case for finite groups. I, II, Ann. of Math. 117 (1983), 383-551. MR 84g:20021a; MR 84g:20021b

[AS] M. Aschbacher and S. D. Smith, A classification of quasithin groups, Amer. Math. Soc. Surveys and Monographs, to appear.

[Ba] B. Baumann, Über endliche Gruppen mit einer zu $L_{2}\left(2^{n}\right)$ isomorphen Faktorgruppe, Proc. Amer. Math. Soc. 74 (1979), 215-222. MR 80g:20024

[Be1] H. Bender, On the uniqueness theorem, Illinois J. Math. 14 (1970), 376-384. MR 41:6959

[Be2] H. Bender, On groups with abelian Sylow 2-subgroups, Math. Z. 117 (1970), 164-176. MR 44:5378

[Be3] H. Bender, Transitive Gruppen gerader Ordnung in denen jede Involution genau einen Punkt festlasst, J. Algebra 17 (1971), 527-554. MR 44:5370

[Be4] H. Bender, Finite groups with dihedral Sylow 2-subgroups, J. Algebra 70 (1981), 216-228. MR 83c:20011b

[Be5] H. Bender, Entwicklungslinien in der Theorie endlicher Gruppen, Jber. d. Dt. Math.-Verein. (1992), 77-123. 
[BG1] H. Bender and G. Glauberman, Characters of finite groups with dihedral Sylow 2-subgroups, J. Algebra 70 (1981), 200-215. MR 83c:20011a

[BG2] H. Bender and G. Glauberman, Local Analysis for the Odd Order Theorem, London Math. Soc. Lecture Notes Series 188, Cambridge U. Press, Cambridge, 1994. MR 96h:20036

[Br1] R. Brauer, On groups whose order contains a prime to the first power. I, II, Amer. J. Math. 64 (1942), 401-440. MR 4:1e MR 4:2a

[Br2] R. Brauer, Blocks of characters and structure of finite groups, Bull. Amer. Math. Soc. (New Series) 1 (1979), 21-38. MR 80f:20013

[BF] R. Brauer and K. A. Fowler, On groups of even order, Ann. of Math. 62 (1955), 565-583. MR 17:580e

[BS] R. Brauer and M. Suzuki, On finite groups of even order whose 2-Sylow subgroup is a quaternion group, Proc. Natl. Acad. Sci. USA 45 (1959), 1757-1759. MR 22:731]

[BSW] R. Brauer, M. Suzuki and G. E. Wall, A characterization of the one-dimensional unimodular groups over finite fields, Illinois J. Math. 2 (1958), 718-745. MR 21:3487]

[Bu1] W. Burnside, Notes on the theory of groups of finite order, Proc. London Math. Soc. 26 (1895), 191-214.

[Bu2] W. Burnside, On a class of groups of finite order, Trans. Cambridge Phil. Soc. 18 (1899), 269-276.

[Bu3] W. Burnside, On some properties of groups of odd order, Proc. London Math. Soc. 32 (1900), 162-185, 257-268.

[Bu4] W. Burnside, On groups of order $p^{\alpha} q^{\beta}$, Proc. London Math. Soc. (2) 1 (1904), 388-392.

[Bu5] W. Burnside, Theory of Groups of Finite Order, Second edition, Cambridge University Press, Cambridge, 1911.

[Ch] C. Chevalley, Sur certains groupes simples, Tôhoku Math. J. 7 (1955), 14-66. MR 17:457c

[Cl] M. J. Collins (ed.), Finite Simple Groups II, Academic Press, London, 1980. MR 82h:20021

[Co1] F. N. Cole, Simple groups from order 201 to order 500, Amer. J. Math. 14 (1892), 378-388.

[Co2] F. N. Cole, Simple groups as far as order 660, Amer. J. Math. 15 (1893), 303-315.

[CG] F. N. Cole and J. W. Glover, On groups whose orders are products of three prime factors, Amer. J. Math 15 (1893), 191-220.

[Da] E. Dade, Lifting group characters, Ann. of Math. 79 (1964), 590-596. MR 28:4023

[DGS] A. Delgado, D. Goldschmidt and B. Stellmacher, Groups and Graphs: New Results and Methods, DMW Seminar Band 6, Birkhäuser Verlag, Basel, 1985. MR 88a:05076

[D1] L. E. Dickson, Linear Groups with an Exposition of the Galois Field Theory, Teubner, Leipzig, 1901.

[D2] L. E. Dickson, Theory of linear groups in an arbitrary field, Trans. Amer. Math. Soc. 2 (1901), 363-394.

[D3] L. E. Dickson, A new system of simple groups, Math. Annalen 60 (1905), 137-150.

[Du] P. Duren (ed.), A Century of Mathematics in America, Part I, American Mathematical Society, Providence, RI, 1988. MR 90a:01064

[E] M. Enguehard, Obstructions et p-groupes de classe 3. Caracterisation des groupes de Ree, Astérisque 142-143 (1986), 3-139. MR 88a:20028

[Fe] W. Feit, Richard D. Brauer, Bull. Amer. Math. Soc. (New Series) 1 (1979), 1-20. MR 80m:01033

[FHT] W. Feit, M. Hall, Jr. and J. G. Thompson, Finite groups in which the centralizer of any non-identity element is nilpotent, Math. Zeit. 74 (1960), 1-17. MR 22:5674

[FT] W. Feit and J. G. Thompson, Solvability of groups of odd order, Pacific J. Math. 13 (1963), 775-1029. MR 29:3538

[Fi] B. Fischer, Groups generated by 3-transpositions, Invent. Math. 13 (1971), 232-246. MR 45:3557

[F] H. Fitting, Beiträge zur Theorie der Gruppen endlicher Ordnung, Jahr. Deutsch. Math. Ver. 48 (1938), 77-141.

[FS] P. Fong and G. M. Seitz, Groups with a $(B, N)$-pair of rank 2. I, II, Invent. Math. 21 (1973), 1-57; Invent. Math. 24 (1974), 191-239. MR 50:7335

[Fr1] F. G. Frobenius, Über auflösbare Gruppen, Sitzungsber. Kön. Preuss. Akad. Wiss. Berlin (1893), 337-345.

[Fr2] F. G. Frobenius, Über auflösbare Gruppen IV, Sitzungsber. Kön. Preuss. Akad. Wiss. Berlin (1901), 1216-1230. 
[GG] R. H. Gilman and R. L. Griess, Finite groups with standard components of Lie type over fields of characteristic two, J. Algebra 80 (1983), 383-516. MR 84g:20024

[Gl1] G. Glauberman, Central elements in core-free groups, J. Algebra 4 (1966), 403-420. MR 34:2681

[Gl2] G. Glauberman, A characteristic subgroup of a p-stable group, Canad. J. Math. 20 (1968), 1101-1135. MR 37:6365

[G13] G. Glauberman, Prime-power factor groups of finite groups, I, Math. Z. 107 (1968), 159172; II, Math. Z. 117 (1970), 46-56. MR 39:298 MR 45:3553

[Gl4] G. Glauberman, On solvable signalizer functors in finite groups, Proc. London Math. Soc. (3) 33 (1976), 1-27. MR 54:5341

[GN] G. Glauberman and R. Niles, A pair of characteristic subgroups for pushing-up finite groups, Proc. London Math. Soc. (3) 46 (1983), 411-453. MR 84g:20042

[Go1] D. M. Goldschmidt, A group-theoretic proof of the $p^{a} q^{b}$-theorem for odd primes, Math. Z. 113 (1970), 373-375. MR 43:2085

[Go2] D. M. Goldschmidt, Solvable signalizer functors on finite groups, J. Algebra 21 (1972), 137-148. MR 45:6913

[Go3] D. M. Goldschmidt, 2-signalizer functors on finite groups, J. Algebra 21 (1972), 321-340. MR 48:2257

[Go4] D. M. Goldschmidt, 2-fusion in finite groups, Ann. of Math. 99 (1974), 70-117. MR 49:407

[Go5] D. M. Goldschmidt, Automorphisms of trivalent graphs, Ann. of Math. 111 (1980), 377406. MR 82a:05052

[G1] D. Gorenstein, Finite Groups, Harper and Row, New York, 1968. MR 38:229

[G2] _ On the centralizers of involutions in finite groups, J. Algebra 11 (1969), 243-277. MR 39:1540

[G3] — The classification of finite simple groups, Bull. Amer. Math. Soc. (New Series) 1 (1979), 43-199. MR 80b:20015

[G4] - Finite Simple Groups: An Introduction to their Classification, Plenum Press, New York, 1982. MR 84j:20002

[G5] - The Classification of Finite Simple Groups, Plenum Press, New York, 1983. MR 86i:20024

[G6] The classification of the finite simple groups, a personal journey: the early years, pp. 447-476 in [Du]. MR 90g:01031

[GH] D. Gorenstein and K. Harada, Finite groups whose 2-subgroups are generated by at most 4 elements, Memoirs Amer. Math. Soc. 147 (1974). MR 51:3290

[GL1] D. Gorenstein and R. Lyons, The local structure of finite groups of characteristic 2 type, Memoirs Amer. Math. Soc. 276 (1983). MR 84g:20025

[GL2] _ A local characterization of some sporadic groups, unpublished typescript.

[GLS1] D. Gorenstein, R. Lyons and R. Solomon, The Classification of the Finite Simple Groups, Number 1, Amer. Math. Soc. Surveys and Monographs 40, \#1 (1994). MR 95m:20014

[GLS2] — The Classification of the Finite Simple Groups, Number 3, Amer. Math. Soc. Surveys and Monographs 40, \#3 (1998). MR 98j:20011

[GW1] D. Gorenstein and J. H. Walter, The characterization of finite groups with dihedral Sylow 2-subgroups, J. Algebra 2 (1965), 85-151, 218-270, 354-393. MR 31:1297a

[GW2] _ The $\pi$-layer of a finite group, Illinois J. Math. 15 (1971), 555-565. MR 44:6812

[GW3] _ Balance and generation in finite groups, J. Algebra 33 (1975), 224-287. MR 50:10051

[Gr] R. L. Griess, The friendly giant, Invent. Math. 69 (1982), 1-102. MR 84m:20024

[GMS] R. L. Griess, U. Meierfrankenfeld and Y. Segev, A uniqueness proof for the Monster, Ann. of Math. 130 (1989), 567-602. MR 90j:20030

[GR] K. W. Gruenberg and J. E. Roseblade, Group Theory: Essays for Philip Hall, Academic Press, London, 1984. MR 85k:20004

[Gu] O. Grün, Beiträge zur Gruppentheorie I, II, Crelle J. 174 (1936), 1-14; J. Reine Angew. Math. 186 (1945), pp. 165-169. MR 10:504c

[Ha] M. Hall, Jr., The Theory of Groups, Macmillan, New York, 1959. MR 21:1996

[H1] P. Hall, A note on soluble groups, J. London Math. Soc. 3 (1928), 98-105.

[H2] P. Hall, A contribution to the theory of groups of prime power order, Proc. London Math. Soc. (2) 36 (1933), 29-95.

[H3] P. Hall, A characteristic property of soluble groups, J. London Math. Soc. 12 (1937), 198-200. 
[H4] P. Hall, On the Sylow systems of a soluble group, Proc. London Math. Soc. (2) 43 (1937), 316-323.

[H5] P. Hall, Theorems like Sylow's, Proc. London Math. Soc. (3) 6 (1956), 286-304. MR 17:1052a

[HH] P. Hall and G. Higman, The p-length of a p-soluble group and reduction theorem for Burnside's problem, Proc. London Math. Soc. (3) 6 (1956), 1-42. MR 17:344b

[Hr1] K. Harada, Groups with nonconnected Sylow 2-subgroups revisited, J. Algebra 70 (1981), 339-349. MR 82i:20023

[Hr2] K. Harada, Michio Suzuki, in Advanced Studies in Pure Mathematics: Groups and Combinatorics, Japan Math. Society, to appear.

[Ho] O. Hölder, Die einfachen Gruppen in ersten und zweiten Hundert der Ordnungszahlen, Math. Annalen 40 (1892), 55-88.

[Ja] Z. Janko, A new finite simple group with abelian 2-Sylow subgroups and its characterization, J. Algebra 3 (1966), 147-186. MR 33:1359

[J1] C. Jordan, Traité des Substitutions et des Équations Algébriques, Gauthier-Villars, Paris, 1870.

[J2] C. Jordan, Recherches sur les substitutions, J. de Math. Pures et Appl. 17 (1872), 345-361.

[KM] K. Klinger and G. Mason, Centralizers of p-subgroups in groups of characteristic 2, p-type, J. Algebra 37 (1975), 362-375. MR 52:10873

[Ms1] G. Mason, Quasithin groups, pp. 181-197 in [Cl]. MR 82h:20021

[Ms2] G. Mason, Quasithin finite groups, unpublished typescript.

[Ma] H. Matsuyama, Solvability of groups of order $2^{a} p^{b}$, Osaka J. Math. 10 (1973), 375-378. MR 48:2243

[McB1] P. P. McBride, Near solvable signalizer functors on finite groups, J. Algebra 78 (1982), 181-214. MR 84g:20049a

[McB2] P. P. McBride, Nonsolvable signalizer functors on finite groups, J. Algebra 78 (1982), 215-238. MR 84g:20049b

[MBD] G. A. Miller, H. F. Blichfeldt and L. E. Dickson, Theory and Applications of Finite Groups, John Wiley and Sons, New York, 1916.

[ML] G. A. Miller and G. H. Ling, Proof that there is no simple group whose order lies between 1092 and 2001, Amer. J. Math. 22 (1900), 13-26.

[Pe1] T. Peterfalvi, Sur la caractérisation des groupes $U_{3}(q)$, pour $q$ impair, J. Algebra 141 (1991), 253-264. MR 93c:20034

[Pe2] T. Peterfalvi, Character Theory for the Odd Order Theorem, London Math. Soc. Lecture Note Series \# 272, Cambridge U. Press, Cambridge, 2000. MR 2001a:20016

[PT] M. Powell and G. Thwaites, On the nonexistence of certain types of subgroups in simple groups, Quart. J. Math. Oxford Series (2) 26 (1975), 243-256. MR 56:466

[Rd] L. Rédei, Ein Satz über die endlichen einfachen Gruppen, Acta Math. 84 (1950), 129-153. MR 13:907d

[Re1] R. Ree, A family of simple groups associated with the simple Lie algebra of type $\left(F_{4}\right)$, Amer. J. Math. 83 (1961), 401-420. MR 24:A2617

[Re2] R. Ree, A family of simple groups associated with the simple Lie algebra of type $\left(G_{2}\right)$, Amer. J. Math. 83 (1961), 432-462. MR 25:2123

[Ro] J. E. Roseblade, Obituary: Philip Hall, Bull. London Math. Soc. 16 (1984), 603-626. MR 86c:01056

[Se] G. M. Seitz, Standard subgroups in finite groups, pp. 41-62 in [Cl]. MR 82h:20021

[So] R. M. Solomon, The B(G)-conjecture and unbalanced groups, pp. 63-87 in [Cl]. MR 82h:20021

[Sp] A. Speiser, Theorie der Gruppen von endlicher Ordnung, Springer-Verlag, Berlin, 1937. MR 82j:20003

[St] R. Steinberg, Variations on a theme of Chevalley, Pacific J. Math. 9 (1959), 875-891. MR 22:79

[S11] B. Stellmacher, A characteristic subgroup of $\Sigma_{4}$-free groups, Israel J. Math. 94 (1996), 367379. MR 97d:20018

[S12] B. Stellmacher, An application of the amalgam method: the 2-local structure of N-groups of characteristic 2-type, J. Algebra 190 (1997), 11-67. MR 98d:20018

[S13] B. Stellmacher, Finite groups all of whose maximal parabolic subgroups are thin and of characteristic 2-type, unpublished typescript. 
[Su1] M. Suzuki, On the finite group with a complete partition, J. Math. Soc. Japan 2 (1950), 165-185. MR 13:907b

[Su2] M. Suzuki, The nonexistence of a certain type of simple groups of odd order, Proc. Amer. Math. Soc. 8 (1957), 686-695. MR 19:248f

[Su3] M. Suzuki, A new type of simple groups of finite order, Proc. Nat. Acad. Sci. U.S.A. 46 (1960), 868-870. MR 22:11038

[Su4] M. Suzuki, On a class of doubly transitive groups, Ann. of Math. 75 (1962), 105-145. MR 25:112

[Su5] M. Suzuki, On a class of doubly transitive groups. II, Ann. of Math. 79 (1964), 514-589. MR 29:144

[Sy] L. Sylow, Théorèmes sur les groupes de substitutions, Math. Ann. 5 (1872), 584-594.

[Sz] G. Szekeres, Determination of a certain family of finite metabelian groups, Trans. Amer. Math. Soc. 66 (1949), 1-43. MR 11:320i

[T1] J. G. Thompson, Normal p-complements for finite groups, Math. Z. 72 (1959/60), 332-354. MR 22:8070

[T2] Nonsolvable finite groups all of whose local subgroups are solvable. I,II,III,IV,V,VI, Bull. Amer. Math. Soc. 74 (1968), 383-437 MR 37:6367; Pacific J. Math. 33 (1970), 451-536 MR 43:2072; Pacific J. Math. 39 (1971), 483-534 MR 47:1933; Pacific J. Math. 48 (1973), 511-592 MR 51:5745. Pacific J. Math. 50 (1974), 215-297; Pacific J. Math. 51 (1974), $573-630$.

[T3] _ Quadratic pairs, Actes du Congrès Intl. des Math. (Nice, 1970), Tome 1, GauthierVillars, Paris, 1971. MR 55:3051

[T4] _ Finite non-solvable groups, pp. 1-12 in GR. MR 85k:20004

[Tm1] F. G. Timmesfeld, Groups generated by root involutions. I, II, J. Algebra 33 (1975), 75134; J. Algebra 35 (1975), 367-441. MR 51:8236

[Tm2] F. G. Timmesfeld, Finite simple groups in which the generalized Fitting-group of the centralizer of some involution is extraspecial, Ann. of Math. 107 (1978), 297-369. MR 81i:20016a

[Ti] J. Tits, Buildings of spherical type and finite BN-pairs, Lecture Notes in Mathematics \# 386, Springer-Verlag, Berlin, 1974. MR 57:9866

[Wa] J. H. Walter, The characterization of finite groups with abelian Sylow 2-subgroups, Ann. of Math. 89 (1969), 405-514. MR 40:2749

[Wi] H. Wielandt, p-Sylowgruppen und p-Faktorgruppen, J. Reine Angew. Math. 182 (1940), 180-193. MR 2:216e

[Y] T. Yoshida, Character-theoretic transfer, J. Algebra 52 (1978), 1-38. MR 58:11095

[Z1] H. J. Zassenhaus, Kennzeichnung endlicher linearer Gruppen, Abh. Math. Sem. Hamburg 11 (1936), 17-40.

[Z2] H. J. Zassenhaus, Über endliche Fastkörper, Abh. Math. Sem. Hamburg 11 (1936), 187-220.

[Z3] H. J. Zassenhaus, The Theory of Groups, Second Edition, Chelsea, New York, 1949. MR 11:77d

Department of Mathematics, The Ohio State University, Columbus, OH 43210

E-mail address: solomon@math.ohio-state.edu 